Pacific Journal of Mathematics

INTEGRATED SEMIGROUPS AND THEIR APPLICATIONS TO 


\section{INTEGRATED SEMIGROUPS AND THEIR APPLICATIONS TO THE ABSTRACT CAUCHY PROBLEM}

Frank Neubrander

This paper is concerned with characterizations of those linear, closed, but not necessarily densely defined operators $A$ on a Banach space $E$ with nonempty resolvent set for which the abstract Cauchy problem $u^{\prime}(t)=A u(t), u(0)=x$ has unique, exponentially bounded solutions for every initial value $x \in D\left(A^{n}\right)$.

Investigating these operators we are led to the class of "integrated semigroups". Among others, this class contains the classes of strongly continuous semigroups and cosine families and the class of exponentially bounded distribution semigroups.

The given characterizations of the generators of these integrated semigroups unify and generalize the classical characterizations of generators of strongly continuous semigroups, cosine families or exponentially bounded distribution semigroups.

We indicate how integrated semigroups can be used studying second order Cauchy problems $u^{\prime \prime}(t)-A_{1} u^{\prime}(t)-A_{2} u(t)=0$, operator valued equations $U^{\prime}(t)=A_{1} U(t)+U(t) A_{2}$ and nonautonomous equations $u^{\prime}(t)=A(t) u(t)$.

1. Introduction. We study integrated semigroups and their connection to the abstract Cauchy problem

$$
u^{\prime}(t)=A u(t) ; \quad u(0)=x
$$

where $A$ is a linear, closed operator on a Banach space $E$ with nonempty resolvent set and domain $D(A)$.

A function $u(\cdot):[0, \infty) \rightarrow D(A)$ with $u(\cdot) \in C^{1}([0, \infty), E)$ and $u(0)=x$ which satisfies (ACP) is called a solution of (ACP).

Studying (ACP), we will introduce the notion of "generators of integrated semigroups". If $A$ is such a generator, then (ACP) is exponentially wellposed in the following sense: there exist an $n \in N$ and constants $M, w$, such that, for all $x \in D\left(A^{n}\right)$, there exists a unique solution $u(\cdot)$ of (ACP) with $|u(t)| \leq M e^{w t}|x|_{n-1}$ for all $t \geq 0$, where $|x|_{n-1}:=|x|+|A x|+\cdots+\left|A^{n-1} x\right|$ denotes the graph norm of the Banach space $\left[D\left(A^{n-1}\right)\right]$ (for a more refined definition of wellposedness, see Def. 3.2). 
We will give several necessary and sufficient conditions on $A$ such that (ACP) is exponentially wellposed.

If possible we will avoid the assumption that $A$ is densely defined. There are many situations where such operators occur. Some examples are given by adjoint operators of generators on nonreflexive Banach spaces (see $\S 6$ ); $L(E)$-valued Cauchy problems for operators $\Gamma B=A_{1} B+B A_{2}$ where $A_{i}$ are unbounded operators on $E$ and $B \in L(E)$ or differential operators in spaces $L^{\infty}(\Omega)$ (see $\S 9$ ). Other nondensely defined operators appear if one studies the reduction of nonautonomous evolution equations $u^{\prime}(t)=A(t) u(t)$ on a Banach space $E$ to an autonomous one on $L^{\infty}\left(R^{+}, L(E)\right)$ (see $\left.\S 9\right)$ or if one studies perturbed Cauchy problems for $u^{\prime}(t)=A u(t)+B u(t)$ where $A$ is a semigroup generator on $E$ (as the Laplacian on $E=C_{0}(\Omega)$, $\Omega \subset R^{n}$ ) and the operator $B$ maps into a "bigger" Banach space $F$ (as $F=C(\Omega)$ ). These problems can be treated using the variation-ofconstants formula

$$
u(t)=T(t) x+\int_{0}^{t} T(t-s) B u(s) d s
$$

which makes sense if the operators $T(t)$ can be extended on $F$. However, the generator of this extended (generally not strongly continuous) semigroup is in many cases no longer densely defined.

In $\S 7$ we will show how integrated semigroups can be used to study the second order abstract Cauchy problem

$$
u^{\prime \prime}(t)=A u(t), \quad u(0)=x, \quad u^{\prime}(0)=y
$$

for linear operators not necessarily generating a strongly continuous cosine family (for a brief introduction to cosine family theory see [11]; for a comprehensive study of the second order Cauchy problem see [10]).

In $\S 8$ we study second order problems with "structural damping" (see [5]). The models investigated are of the form

$$
u^{\prime \prime}(t)-A u^{\prime}(t)-\left(a A^{2}+b A+c I\right) u(t)=0, \quad u(0)=x, \quad u^{\prime}(0)=y
$$

where $A$ is a linear operator with nonempty resolvent set. We will show that these damped second order Cauchy problems are exponentially wellposed whenever the resolvent of $A$ is polynomially bounded on a region determined by the parameter $a$. In case that $A$ generates a strongly continuous semigroup, explicit expressions for the solutions are given. 
In $\S 9$ we indicate how integrated semigroups can be used studying $L(E)$-valued Cauchy problems $U^{\prime}(t)=A_{1} U(t)+U(t) A_{2}, U(0)=X \in$ $L(E)$; evolution equations $u^{\prime}(t)=A(t) u(t), u(s)=x$ and Cauchy problems connected with differential operators $p\left(d_{1} / d x_{1}, \ldots, d_{n} / d x_{n}\right)$ on $L^{p}\left(R^{n}\right)(1 \leq p \leq \infty)$ or $C_{0}\left(R^{n}\right)$.

2. Existence and uniqueness of solutions. We recall that a densely defined operator $A$ is a semigroup generator if and only if there exist constants $M, w$ such that every real number $\mu$ with $\mu>w$ is an element of the resolvent set of $A$ and

$$
\left|\frac{1}{k !}(R(\mu, A))^{(k)}\right|=\left|R(\mu, A)^{k+1}\right| \leq M\left(\frac{1}{\mu-w}\right)^{k+1}
$$

for every $k \in N_{0}$ and $\mu>w$ (here and in the sequel $f(\mu)^{(k)}$ stands for $\left.d^{k} / d \mu^{k} f(\mu)\right)$.

If $A$ is a linear operator with spectrum contained in a left halfplane, then the growth assumptions (2.1) on the resolvent which imply existence and uniqueness of solutions of (ACP) for all $x \in D(A)$ are the strongest possible ones; i.e., there is no nontrivial operator satisfying $|R(\mu, A)| \leq M(1 /(\mu-w))^{k}$ for all $\mu>w$ and some $k>1$ (this follows from the resolvent identity $x=\mu R(\mu, A) x-R(\mu, A) A x)$.

Uniqueness of solutions of (ACP) requires much weaker growth assumptions on the resolvent. It was shown by Ljubic that if $A$ is a linear operator on a Banach space $E$ such that the resolvent $R(\mu, A)$ exists for all $\mu>w$ and satisfies

$$
\lim _{\mu \rightarrow \infty} \frac{1}{\mu} \log |R(\mu, A)|=0,
$$

then (ACP) has at most one solution for every $x \in E$. The proof of (2.2) given in [28], 4.1 does not make use of the assumption that $A$ is densely defined; hence (2.2) holds for nondensely defined operators.

There is a wide gap between (2.1) and (2.2). As we will see, growth conditions not as strong as (2.1) and not as weak as (2.2) imply existence and uniqueness of solutions of (ACP) for a large set of initial data. Furthermore we will show that existence and uniqueness of solutions always imply that the solutions depend "continuously" on the initial data. Hence (ACP) is wellposed under much weaker growth assumptions on the resolvent than those stated in (2.1).

If a linear operator $A$ has a resolvent in a right half plane and if $u(\cdot)$ is an exponentially bounded solution of the corresponding (ACP) with $u(0)=x$, then the resolvent $R(\mu, A) x$ is the Laplace transform 
of $u(\cdot)$, i.e.: $R(\mu, A) x=\int_{0}^{\infty} e^{-\mu t} u(t) d t$ (see Lemma 4.6). Therefore it is natural to try to construct a solution $u(\cdot)$ as the inverse Laplace transform of $R(\mu, A) x$; i.e.:

$$
u(t)=\frac{1}{2 \pi i} \int_{p-i \infty}^{p+i \infty} e^{\mu t} R(\mu, A) x d \mu .
$$

In the following two propositions we recall growth assumptions on $R(\mu, A) x$ respectively $R(\mu, A)$ which ensure that the inverse Laplace transform exists, is differentiable and a solution of (ACP). We need the following lemma (see [26]).

LEMMA 2.1. Every linear operator $A$ with nonempty resolvent set is closed. If, in addition, $A$ is densely defined, then $D\left(A^{n}\right)$ is dense in $E$ for every $n \in N$ and $D\left(A^{m}\right)$ is dense in the Banach space $\left[D\left(A^{n}\right)\right]$ for every $m \geq n$.

Proposition 2.2. Let $A$ be a linear operator on a Banach space $E$ such that, for $a w>0$, every $\mu \in C$ with $\operatorname{Re} \mu>w$ is in the resolvent set of $A$. Let $x \in D\left(A^{n}\right)$ for some $n \geq 3$. If there is a constant $M(x)$ such that $\left|R(\mu, A) A^{n} x\right| \leq M(x)$ for $\operatorname{Re} \mu>w$, then (ACP) has a unique solution $u(\cdot)$ with $u(0)=x$ and $|u(t)| \leq C e^{p t}|x|_{n-1}$ for all $t \geq 0$.

Proof. Let $p>w$. From $\left|R(\mu, A) A^{n} x\right| \leq M(x)$ it follows that

$$
v(t):=\frac{1}{2 \pi i} \int_{p-i \infty}^{p+i \infty} e^{\mu t} \mu^{-n} R(\mu, A) A^{n} x d \mu
$$

is a continuously differentiable function with $|v(t)| \leq C(x) e^{p t}$ and $v(0)=0$. By $R(\mu, A) x=\mu^{-1}(R(\mu, A) A x-x)$ we obtain $\left|R(\mu, A) A^{n-1} x\right|$ $\leq C(x)$. By the closedness of $A$ (Lemma 2.1),

$$
v^{\prime}(t)=A \frac{1}{2 \pi i} \int_{p-i \infty}^{p+i \infty} e^{\mu t} \mu^{1-n} R(\mu, A) A^{n-1} x d \mu .
$$

Define $u(t):=\sum_{k=0}^{n-1} 1 /(k !) t^{k} A^{k} x+v(t)$. Clearly, $u(\cdot)$ is continuously differentiable, $u(0)=x$ and $|u(t)| \leq C_{x} e^{p t}|x|_{n-1}$. By the equation $A R(\mu, A) A^{n} x=\mu R(\mu, A) A^{n} x-A^{n} x$ we see that

$$
\frac{1}{2 \pi i} \int_{p-i \infty}^{p+i \infty} e^{\mu t} \mu^{-n} A R(\mu, A) A^{n} x d \mu
$$

exists. Therefore $u(t) \in D(A)$ and an easy computation shows that $u^{\prime}(t)=A u(t)$. Hence $u(\cdot)$ is a solution of (ACP) with $u(0)=x$. 
The following proposition can be found in [18], 1.3 (for a generalization, see [4]). Notice that the statements of Propositions 2.2 and 2.3 are valid for nondensely defined operators.

Proposition 2.3. Let $A$ be a linear operator on a Banach space $E$. If there are constants $w, M$ such that $R(\mu, A)$ exists and satisfies

$$
|R(\mu, A)| \leq M(1+|\mu|)^{k} \quad \text { for some }-1 \leq k \in N
$$

for all $\mu \in C$ with $\operatorname{Re} \mu>w$, then (ACP) has a unique solution $u(\cdot)$ for every $x \in D\left(A^{k+3}\right)$ such that $|u(t)| \leq M_{p} e^{p t}|x|_{k+3}$ for $p>w$. If, for some $-1 \leq \alpha<0,|R(\mu, A)| \leq M(1+|\operatorname{Im} \mu|)^{\alpha}$ for all $\mu \in C$ with $\operatorname{Re} \mu>w$, then (ACP) has a unique solution $u(\cdot)$ for every $x \in D\left(A^{2}\right)$ and, for $p>w,|u(t)| \leq M e^{p t}|x|_{2}$.

We now give an example which shows that for every $n \in N$ there is an operator $A$ for which (ACP) has unique solutions for every $x \in$ $D\left(A^{n}\right)$ but not for every $x \in D\left(A^{n-1}\right)$ and that (2.3) restricted to real $\mu$ is not sufficient to guarantee existence of solutions for $x \in D\left(A^{k+3}\right)$.

Proposition 2.4. For every $n \geq 1$ there exists a densely defined linear operator $A$ on a Banach (Hilbert) space $E$ such that

(a) the resolvent $R(\mu, A)$ exists for $\operatorname{Re} \mu>0$ and satisfies $|R(\mu, A)| \leq$ $\left(2^{n}-1\right) / \mu$ for all real $\mu>0$ and

(b) the abstract Cauchy problem has unique solutions for every $x \in$ $D\left(A^{n}\right)$ but not for every $x \in D\left(A^{n-1}\right)$.

Proof. For $n=1$, the statements are obvious. Let $n \geq 2$ and let $A$ generate a nonholomorphic contraction semigroup $(T(t))$ on a Banach (Hilbert) space $E$. Let $E^{n}$ be the space $E \times \cdots \times E$ endowed with the maximum (Hilbert) norm. Then

$$
B:=\left[\begin{array}{ccccc}
A & A & 0 & \cdot & 0 \\
0 & A & A & . & . \\
0 & 0 & A & \cdot & 0 \\
. & . & \cdot & . & A \\
0 & . & . & 0 & A
\end{array}\right]
$$


is a densely defined, closed operator on $E^{n}$ with $D\left(B^{j}\right)=D\left(A^{j}\right)^{n}$ for every $j \in N$. The resolvent sets of $A$ and $B$ are equal,

$$
\begin{gathered}
R(\mu, B)=\left[\begin{array}{cccc}
R(\mu, A) & A R(\mu, A)^{2} & \cdots & A^{n-1} R(\mu, A)^{n} \\
0 & R(\mu, A) & \cdots & \cdot \\
\cdot & 0 & \cdots & \cdot \\
\cdot & \cdot & \cdot & \cdot \\
\cdot & \cdot & 0 & A R\left(\mu, A^{2}\right) \\
0 & \cdot & 0 & R(\mu, A)
\end{array}\right], \quad \text { and } \\
|R(\mu, B)| \leq \sum_{k=0}^{n-1}\left|A^{k} R(\mu, A)^{k+1}\right| \\
\leq \frac{1}{\mu} \sum_{k=0}^{n-1}|\mu R(\mu, A)-I|^{k} \\
\leq\left(2^{n}-1\right) \frac{1}{\mu}
\end{gathered}
$$

for all $\mu>0$. However, for $n \geq 2, B$ does not generate a strongly continuous semigroup on $E^{n}$. We prove this by showing that

$$
u^{\prime}(t)=B u(t), \quad u(0)=\left(u_{0}, \ldots, u_{n-1}\right)
$$

has unique solutions for every $u(0) \in D\left(B^{n}\right)$ but not for every $u(0) \in$ $D\left(B^{n-1}\right)$. Suppose $(*)$ has a solution $\left(u_{0}(t), \ldots, u_{n-1}(t)\right)$. Then $u_{n-1}(t)$ is a solution of $u_{n-1}^{\prime}(t)=A u_{n-1}(t), u_{n-1}(0)=u_{n-1}$. Hence $u_{n-1}$ has to be in $D(A)$ and $u_{n-1}(t)=T(t) u_{n-1}$. (From $u_{n-1} \in D(A)$ we conclude that $(*)$ has a solution with initial value $\left(0, \ldots, 0, u_{n-1}\right)$; therefore $(*)$ has a solution with initial value $\left(u_{0}, \ldots, u_{n-2}, 0\right)$. The same argument as above gives then that $u_{n-2}$ has to be in $D(A)$. Repeating this procedure we obtain that $u_{i} \in D(A)$ for all $0 \leq i \leq n-1$.) The function $u_{n-2}(t)$ is a solution of

$$
u_{n-2}^{\prime}(t)=A u_{n-2}(t)+A u_{n-1}(t)=A u_{n-2}(t)+A T(t) u_{n-1} .
$$

By the variation-of-constants formula

$$
\begin{aligned}
u_{n-2}(t) & =T(t) u_{n-2}+\int_{0}^{t} T(t-s) A T(s) u_{n-1} d s \\
& =T(t) u_{n-2}+t A T(t) u_{n-1}
\end{aligned}
$$

by $u_{n-2} \in D(A)$, and by the assumption that the semigroup is nonholomorphic we conclude that $u_{n-1}$ has to be in $D\left(A^{2}\right)$. Proceeding in this manner we obtain that the initial value of every strong 
solution of $(*)$ is in $D(A) \times \cdots \times D\left(A^{n}\right)$. On the other hand, if $\left(u_{0}, \ldots, u_{n-1}\right) \in D(A) \times \cdots \times D\left(A^{n}\right)$, then $\left(u_{0}(t), \ldots, u_{n-1}(t)\right)$ where

$$
u_{i}(t)=\sum_{j=0}^{n-1-i} \frac{1}{j !} t^{j} T(t) A^{j} u_{i+j}
$$

defines a solution of $(*)$. The uniqueness follows from (2.2) and $|R(\mu, B)| \leq M / \mu$.

3. Continuous dependence of solutions upon the data. It is well known that existence and uniqueness of solutions of (ACP) implies continuous dependence of the solutions from the initial data. In this section we will examine the continuous dependence of the solutions which follows from existence and uniqueness of solutions of (ACP) for $x \in D\left(A^{n}\right)$.

THEOREM 3.1. Let $A$ be a linear operator on a Banach space $E$ with nonempty resolvent set. If (ACP) has unique solutions $u(\cdot)$ for every $x \in D\left(A^{n}\right)$, then the following $n$ notions of continuous dependence of the solutions from the initial data hold.

(i) If $\left(u_{k}(\cdot)\right)_{k \in N}$ is a sequence of solutions of (ACP) with $u_{k}(0) \rightarrow 0$ in $\left[D\left(A^{n-1}\right)\right]$, then the solutions $u_{k}(\cdot)$ converge to zero uniformly on compacts of $R^{+}$.

(ii) If $1 \leq j \leq n-1$ and if $\left(u_{k}(\cdot)\right)_{k \in N}$ is a sequence of solutions of $(\mathrm{ACP})$ with $u_{k}(0) \rightarrow 0$ in $\left[D\left(A^{n-j-1}\right)\right]$, then the $j$-times integrated solutions $f_{k}(\cdot)$,

$$
f_{k}(t):=\int_{0}^{t} \frac{1}{(j-1) !}(t-s)^{j-1} u_{k}(s) d s,
$$

converge to zero uniformly on compacts of $R^{+}$.

(iii) There exists a locally bounded function $p(\cdot)$ such that for all $0 \leq j \leq n-1$ and $x \in D\left(A^{n}\right)$, the $j$-times integrated solutions fulfill $|f(t)| \leq p(t)|x|_{n-j-1}$.

Proof. We assume, without loss of generality, that 0 is in the resolvent set of $A$.

(i): Define an operator $S$ from the Banach space $\left[D\left(A^{n}\right)\right]$ into the Fréchet space $C\left(R^{+},[D(A)]\right)$ by $x \rightarrow u(\cdot)$. The operator $S$ is everywhere defined, closed and hence continuous. If $\left(u_{k}(\cdot)\right)$ is a sequence of solutions with $x_{k}:=u_{k}(0) \rightarrow 0$ in $\left[D\left(A^{n-1}\right)\right]$, then $v_{k}(\cdot):=A^{-1} u_{k}(\cdot)=$ $S\left(A^{-1} x_{k}\right)$ converges to zero in $[D(A)]$ because $A^{-1} x_{k}$ converges to zero 
in $\left[D\left(A^{n}\right)\right]$. Hence $u_{k}(\cdot)=A v_{k}(\cdot)$ converges to zero in $E$ uniformly in $t$ on compacts of $R^{+}$.

(ii) for $j=1$ : Let $v_{k}(\cdot)$ be a sequence of solutions of (ACP) such that $v_{k}(0) \rightarrow 0$ in $\left[D\left(A^{n-2}\right)\right]$. Then $w_{k}(\cdot)=A^{-1} v_{k}(\cdot)$ is a sequence of solutions of (ACP) with $w_{k}(0) \rightarrow 0$ in $\left[D\left(A^{n-1}\right)\right]$. By (i), $w_{k}(\cdot) \rightarrow 0$ uniformly on compacts. By the continuity of the functions $A v_{k}(\cdot)$ and the closedness of $A$ we obtain $v_{k}(t)-v_{k}(0)=A \int_{0}^{t} v_{k}(s) d s$. Applying $A^{-1}$ on both sides we get statement (ii) for $j=1$.

(ii): Let $v_{k}(\cdot)$ be a sequence of solutions of (ACP) such that $v_{k}(0) \rightarrow$ 0 in $\left[D\left(A^{n-j-2}\right)\right]$ where $1 \leq j \leq n-2$. Then $w_{k}(\cdot)=A^{-1} v_{k}(\cdot)$ is a sequence of solutions of (ACP) with $w_{k}(0) \rightarrow 0$ in $\left[D\left(A^{n-j-1}\right)\right]$. Suppose that statement (ii) holds for some $j$. Then the $j$-times integrated solutions

$$
\int_{0}^{t} \frac{1}{(j-1) !}(t-s)^{j-1} w_{k}(s) d s
$$

converge to zero uniformly on compacts. By the continuity of the functions $A v_{k}(\cdot)$ and the closedness of $A$ we obtain $v_{k}(t)-v_{k}(0)=$ $A \int_{0}^{t} v_{k}(s) d s$ and hence, by integrating both sides $j$-times and applying $A^{-1}$ on both sides we obtain the following equation which implies statement (ii) for $j+1$ :

$$
\begin{aligned}
& \int_{0}^{t} \frac{1}{(j-1) !}(t-s)^{j-1} w_{k}(s) d s-\frac{1}{j !} t^{j-1} w_{k}(0) \\
& =\int_{0}^{t} \frac{1}{j !}(t-s)^{j-1} v_{k}(s) d s
\end{aligned}
$$

(iii): Define an operator from $D\left(A^{n}\right)$ into $E$ by $S(t) x:=f(t)$ where $f(\cdot)$ is the $j$-times integrated solution $u(\cdot)$ with initial value $x$. From (i) and (ii) it follows that for any compact set $I$ there exists a constant $M_{I}$ such that for all $x \in D\left(A^{n}\right)$ and $t \in I$ we have $|f(t)|=|S(t) x| \leq$ $M_{I}|x|_{n-j-1}=M_{I}|u(0)|_{n-j-1}$. Hence there exists a locally bounded function $p_{j}(\cdot)$ such that $|f(t)| \leq p_{j}(t)|x|_{n-j-1} \leq p(t)|x|_{n-j-1}$ where $p(t):=\max _{j}\left\{p_{j}(t)\right\}$.

Following Hadamard [12] we call a Cauchy problem wellposed if solutions exist for sufficiently many initial data and depend continuously on them in the sense that the zero-convergence of the initial functions and their derivatives up to a certain order implies the zeroconvergence of the solutions in the topology of uniform convergence on compact intervals.

In the following definition we do not specify what precisely it means to have solutions for "sufficiently many initial data". Considering the 
various paths leading to abstract Cauchy problems it does not seem to be "natural" to restrict a discussion of (ACP) to densely defined operators $A$. In applications where $A$ has nondense domain, it is assumed that one checks that the subspaces $D\left(A^{n}\right)$ are large enough to obtain meaningful results.

Definition 3.2. Let $A$ be a closed linear operator on a Banach space $E$. (ACP) is called ( $n, k)$-wellposed (or just wellposed), if there exist an $1 \leq n \in N$, and $k \in N$ with $0 \leq k \leq n$ and a locally bounded function $p(\cdot)$ such that, for all $x \in D\left(A^{n}\right)$, there exists a unique solution $u(\cdot)$ of (ACP) with $|u(t)| \leq p(t)|x|_{k}$ for all $t \geq 0$.

If, in addition, we can choose $p(t)=M e^{w t}$, then (ACP) is called exponentially $(n, k)$-wellposed.

With this terminology, we obtain from Theorem 3.1 the following corollary (see also [29], 2.2).

COROLlARY 3.3. Let $A$ be a linear operator with nonempty resolvent set. If (ACP) has unique solutions $u(\cdot)$ for every $x \in D\left(A^{n}\right)$ then (ACP) is $(n, n-1)$-wellposed.

4. Generators of integrated semigroups and exponentially wellposed Cauchy problems. For densely defined operators, Cauchy problems which are wellposed in a sense similar to Definition 3.2, were investigated by Sova [30], Miyadera-Oharu-Okazawa [21], and Sanekata [29]. We will summarize and extend some of their results in Theorem 4.6.

Before doing this we will add one more concept to the discussion of wellposed Cauchy problems: the concept of integrated semigroups (by a different approach, once integrated, positive semigroups were introduced in [1]). The concept of integrated semigroups provides us with easy to handle characterizations of exponentially wellposed Cauchy problems and enables us to extend known wellposedness results to cases where the operator $A$ has nondense domain.

Definition 4.1. Let $A$ be a linear operator on a Banach space $E$. If there exists an $n \in N$, constants $M, w$ and a strongly continuous family $(S(t))$ in $L(E)$ with $|S(t)| \leq M e^{w t}$ for all $t \geq 0$ such that $R(\mu, A)$ exists and is given by

$$
R(\mu, A) x=\mu^{n-1} \int_{0}^{\infty} e^{-\mu t} S(t) x d t
$$

for $x \in E$ and $\mu>w$, then $A$ is called the generator of the $(n-1)$-times integrated semigroup $(S(t))$. 
If $A$ generates $(S(t))$, then $(S(t))$ is uniquely determined by $A$; i.e., if

$$
R(\mu, A) x=\mu^{n-1} \int_{0}^{\infty} e^{-\mu t} S_{1}(t) x d t=\mu^{n-1} \int_{0}^{\infty} e^{-\mu t} S_{2}(t) x d t
$$

then, by the uniqueness of the Laplace transform, $S_{1}(\cdot)=S_{2}(\cdot)$.

Integrating by parts one sees that if $A$ generates a $j$-times integrated semigroup ( $j \geq 0$ ), then $A$ generates also a $k$-times integrated semigroup for all $k>j$.

It is well known that if the Laplace transform of a continuous function exists for some $\mu_{0} \in C$, then it exists for all complex numbers $\mu$ with $\operatorname{Re} \mu>\operatorname{Re} \mu_{0}$. Hence, if $A$ is the generator of an integrated semigroup $(S(t))$, then $R(\mu, A) x=\mu^{n-1} \int_{0}^{\infty} e^{-\mu t} S(t) x d t$ for all $x \in E$ and $\mu \in C$ with $\operatorname{Re} \mu>w$.

The connection between generators of integrated semigroups and exponentially wellposed abstract Cauchy problems is given through the following theorem.

THEOREM 4.2. Let $A$ be a linear operator on a Banach space $E$ with nonempty resolvent set.

(a) If $A$ generates an ( $n-1)$-times integrated semigroup, then (ACP) is exponentially $(n, n-1)$-wellposed.

(b) If $A$ is densely defined and if (ACP) is exponentially $(n, n-1)-$ wellposed, then $A$ generates an $(n-1)$-times integrated semigroup.

Proof of (b). Let $x \in D\left(A^{n}\right)$. Then there is a unique solution of (ACP) satisfying $|u(t)| \leq M e^{w t}|x|_{n-1}$. For $\mu$ in the resolvent set of $A$, the function $w(t)=R(\mu, A) u(t)$ is a solution of (ACP) with $|w(t)| \leq$ $M^{\prime} e^{w t}|x|_{n-2}$. Let $v(t)=\int_{0}^{t} u(s) d s$ be the once integrated solution. Then $v(t)=\mu \int_{0}^{t} w(s) d s-w(t)+R(\mu, A) x$. Hence $|v(t)| \leq C e^{c t}|x|_{n-2}$ for suitable constants $C$ and $c$. By induction we obtain that the $(n-1)$ times integrated solutions

$$
f(t)=\int_{0}^{t} \frac{1}{(n-2) !}(t-s)^{n-2} u(s) d s
$$

are exponentially bounded.

For $t \geq 0$ define $S(t): D\left(A^{n}\right) \rightarrow E$ by $S(t) x=f(t)$ where $f(\cdot)$ is the unique $(n-1)$-times integrated solution of (ACP) with initial value $x \in D\left(A^{n}\right)$. By Lemma 2.1, the set $D\left(A^{n}\right)$ is dense in $E$. Hence the linear operators $S(t)$ have a unique extension on $E$, denoted by the 
same symbol. From $|S(t)| \leq M e^{w t}$ for all $t \geq 0$ we conclude that $(S(t))$ is strongly continuous. For $\operatorname{Re} \mu>w$ define a bounded linear operator $R(\mu)$ by $R(\mu) x:=\mu^{n-1} \int_{0}^{\infty} e^{-\mu t} S(t) x d t$. Let $x \in D\left(A^{n}\right)$. By the closedness of $A$, one obtains that $S(t) x \in D(A)$ for every $t \geq 0$ and

$$
A S(t) x=\frac{d}{d t} S(t) x-\frac{1}{(n-2) !} t^{n-2} x .
$$

Integrating by parts one shows easily that

$$
\begin{aligned}
A\left(\mu^{n-1} \int_{0}^{T} e^{-\mu t} S(t) x d t\right)= & \mu^{n-1} e^{-\mu T} S(T) x+\mu^{n} \int_{0}^{T} e^{-\mu t} S(t) x d t \\
& -\mu^{n-1} \int_{0}^{T} e^{-\mu t} \frac{1}{(n-2) !} t^{n-2} x d t
\end{aligned}
$$

Hence, for every $x \in D\left(A^{n}\right), R(\mu) x \in D(A)$ and $(\mu-A) R(\mu) x=x$. Now let $x \in E$ and $x_{k} \in D\left(A^{n}\right)$ with $x_{k} \rightarrow x$. Then $A R(\mu) x_{k}=$ $\mu R(\mu) x_{k}-x_{k} \rightarrow \mu R(\mu) x-x$ and $R(\mu) x_{k} \rightarrow R(\mu) x$. Therefore $R(\mu) x \in D(A)$ for $x \in E$ and $A R(\mu) x=\mu R(\mu) x-x$ or $(\mu-A) R(\mu) x=$ $x$. Hence $(\mu-A)$ is onto for every $\mu \in C$ with $\operatorname{Re} \mu>w$. Suppose there is a $y \in D(A)$ with $A y=\mu y$ where $\operatorname{Re} \mu>w$. Then $u(t)=e^{\mu t} y$ is a solution of (ACP) and $y \in D\left(A^{n}\right)$. But

$$
S(t) y=\left(\mu^{1-n} e^{\mu t}-\sum_{j=0}^{n-2} \mu^{1+j-n} \frac{1}{j !} t^{j}\right) y
$$

and hence $|S(t) y| \geq M e^{w t}|y|$ for large $t$ which is impossible. Therefore $\mu$ is in the resolvent set of $A$ and $R(\mu, A)=R(\mu)$.

Proof of (a). If $A$ generates an integrated semigroup, then, by (2.2), by $|S(t)| \leq M e^{w t}$ and by $R(\mu, A) x=\mu^{n-1} \int_{0}^{\infty} e^{-\mu t} S(t) x d t$ we obtain that every solution of (ACP) is unique. The rest of the proof is based on the following lemmas:

LEMMA 4.3. Let $A$ be the generator of an integrated semigroup $(S(t))$. Then $S(t) A x=A S(t) x$ for all $x \in D(A)$.

Proof. Let $x \in D(A)$. Then $R(\mu, A) x=R\left(\mu_{0}, A\right) R(\mu, A)\left(\mu_{0}-A\right) x$. Hence

$$
\int_{0}^{\infty} e^{-\mu t} S(t) x d t=\int_{0}^{\infty} e^{-\mu t} R\left(\mu_{0}, A\right) S(t)\left(\mu_{0}-A\right) x d t
$$

By the uniqueness of the Laplace transform we obtain

$$
S(t) x=R\left(\mu_{0}, A\right) S(t)\left(\mu_{0}-A\right) x \quad \text { or } \quad S(t) A x=A S(t) x .
$$


LEMMA 4.4. Let $A$ be the generator of an $(n-1)$-times integrated semigroup $(S(t))$. Then $S(\cdot) x$ is continuously differentiable for every $x \in D(A), S(0)=0$, and $(d / d t) S(t) x=S(t) A x+t^{n-2} x /(n-2) !$.

Proof. Let $x \in D(A)$. Then

$$
\begin{gathered}
\mu^{n} \int_{0}^{\infty} e^{-\mu t} \frac{1}{(n-1) !} t^{n-1} x d t=x=R(\mu, A)(\mu-A) x \\
=\mu^{n}\left[\int_{0}^{\infty} e^{-\mu t}\left(S(t) x-\int_{0}^{t} S(s) A x d s\right) d t\right]
\end{gathered}
$$

Hence, by the uniqueness of the Laplace transform, we obtain

$$
S(t) x=\int_{0}^{t} S(s) A x d s+\frac{1}{(n-1) !} t^{n-1} x .
$$

The proof of Theorem 4.2 (a) follows from the following observations: Let $x \in D\left(A^{n}\right)$. Define

$$
v(t):=S(t) A^{n-1} x+\sum_{k=0}^{n-2} \frac{1}{k !} t^{k} A^{k} x .
$$

Then $v(0)=x, v(t) \in D(A)$ for $t \geq 0,|v(t)| \leq M e^{w t}|x|_{n-1}$ and $v^{\prime}(t)=A v(t)$. Hence, (ACP) is exponentially wellposed.

If $A$ generates an ( $n-1)$-times integrated semigroup, then it can be shown by using the resolvent identity

$$
R(\mu, A) x=\mu^{-n} R(\mu, A) A^{n} x+\sum_{k=0}^{n-1} \mu^{-(k+1)} A^{k} x,
$$

the identity (4.1) and Phragmen's Representation Theorem (see [24]) that, for all $x \in D\left(A^{n}\right)$, the unique solution $v(\cdot)$ of (ACP) is given by the representation formula

$$
v(t)=\lim _{\mu \rightarrow \infty} \mu \sum_{k=1}^{\infty}(-1)^{k-1} \frac{1}{(k-1) !} e^{k \mu t} R(k \mu, A) x .
$$

In the following theorem several characterizations of densely defined generators of integrated semigroups are given. Statement (f) of Theorem 4.5 coincides with Sova's characterization of densely defined operators for which (ACP) is exponentially wellposed (see [30]). A complex version of Theorem 4.5 (e) is due to Sanekata [29]. To prove Theorem 4.5 we extend Kisynski's ([15], p. 358) and Fattorini's 
([9], Theorem 2.1.1) proof of the Hille-Yosida theorem. As we will see in the following sections, the practically useful characterizations are (b), (c) and (d), whereas it is almost impossible to check the theoretically important growth conditions (e) and (f) in concrete examples. For $n=1$, Theorem 4.6 reduces by $R(\mu, A)^{(k)}=(-1)^{k} k ! R(\mu, A)^{k+1}$ to the classical characterizations of generators of strongly continuous semigroups.

THEOREM 4.5. Let $A$ be a densely defined linear operator on a Banach space $E$. Then the following statements are equivalent:

(a) (ACP) is exponentially ( $n, n-1)$-wellposed.

(b) $A$ is closed and there exists a dense subspace $D$ of $E$ such that, for every $x \in D$, (ACP) has a solution. There exist $M, w$ such that for every solution $u(\cdot)$ with $u(0) \in D$, the $(n-1)$-times integrated solution $f(\cdot)$ satisfy $|f(t)| \leq M e^{w t}|u(0)|$ for all $t \geq 0$.

(c) A generates an $(n-1)$-times integrated semigroup.

(d) There exist $M, w>0$ and a family $(T(t))$ in $L\left(\left[D\left(A^{n-1}\right)\right], E\right)$ with $T(\cdot) x \in C\left(R^{+}, E\right)$ and $|T(t) x| \leq M e^{w t}|x|_{n-1}$ for all $x \in D\left(A^{n-1}\right)$ such that $R(\mu, A)$ exists and is given by $R(\mu, A) x=\int_{0}^{\infty} e^{-\mu t} T(t) x d t$ for all $\mu \in C$ with $\operatorname{Re} \mu>w$ all $x \in D\left(A^{n-1}\right)$.

(e) There exist $M, w>0$ such that $R(\mu, A)$ exists and satisfies $\left|R(\mu, A)^{k} x\right| \leq M(\mu-w)^{-k}|x|_{n-1}$ for all $\mu>w, k \in N$, and all $x \in$ $D\left(A^{n-1}\right)$.

(f) There exist $M, w>0$ such that $R(\mu, A)$ exists and satisfies

$$
\left|\frac{1}{k !}\left(\mu^{1-n} R(\mu, A)\right)^{(k)}\right| \leq M\left(\frac{1}{\mu-w}\right)^{k+1}
$$

for all $\mu>w$ and $k \in N_{0}$.

Proof. The equivalence of (a) and (c) was proved in Theorem 4.2. The proof of the implication (b) $\rightarrow$ (c) is the same as the proof of (a) $\rightarrow\left(\right.$ c), except that the operators $S(t)$ are defined on $D^{\prime}=\{x \in E$ : (ACP) has a solution $\}$ and not on $D\left(A^{n}\right)$. We prove the implications $(c) \rightarrow(d),(f) \rightarrow(b)$ and (e) $\rightarrow$ (b). The implications (d) $\rightarrow$ (e) and (c) $\rightarrow$ (f) are obvious.

To prove the implication (c) $\rightarrow$ (d) we need the following lemma.

LEMMA 4.6. Let $A$ be a linear operator with nonempty resolvent set. If (ACP) has a solution $u(\cdot)$ with $u(0)=x$ such that $|u(t)| \leq C e^{p t}$ for some constants $C, p$, then, for every $\mu$ in the resolvent set of $A$ with $\operatorname{Re} \mu>p$, we have $R(\mu, A) x=\int_{0}^{\infty} e^{-\mu t} u(t) d t$. 
Proof. By the closedness of $A$ (Lemma 2.1) we obtain

$$
\mu \int_{0}^{\infty} e^{-\mu t} u(t) d t-u(0)=\int_{0}^{\infty} e^{-\mu t} u^{\prime}(t) d t=A \int_{0}^{\infty} e^{-\mu t} u(t) d t
$$

Therefore, $(\mu-A) \int_{0}^{\infty} e^{-\mu t} u(t) d t=x$.

Proof of $(\mathrm{c}) \rightarrow(\mathrm{d})$. If $A$ generates an $(n-1)$-times integrated semigroup, then $R(\mu, A)$ exists for every $\mu \in C$ with $\operatorname{Re} \mu>w$. By Theorem 4.2, for every $x \in D\left(A^{n}\right)$ there is a unique solution of (ACP) with $u(0)=x$ and $|u(t)| \leq M e^{w t}|x|_{n-1}$. For $t \geq 0$ define a linear operator on $D\left(A^{n}\right)$ by $T(t) x:=u(t)$. By Lemma $2.1, D\left(A^{n}\right)$ is dense in $\left[D\left(A^{n-1}\right)\right]$. Hence $T(t)$ can be extended to $\left[D\left(A^{n-1}\right)\right]$ such that the extension, which will be denoted by the same symbol, is in $L\left(\left[D\left(A^{n-1}\right)\right], E\right)$. From $|T(t) x| \leq M e^{w t}|x|_{n-1}$ we conclude that $t \rightarrow T(t) x$ is continuous for $x \in D\left(A^{n-1}\right)$. From Lemma 4.6 we get that $R(\mu, A) x=\int_{0}^{\infty} e^{-\mu t} T(t) x d t$ for $x \in D\left(A^{n}\right)$ and $\mu \in C$ with $\operatorname{Re} \mu>w$. But both sides of the last equation define operators in $L\left(\left[D\left(A^{n-1}\right)\right], E\right)$ which coincide on $D\left(A^{n}\right)$ which is dense in $\left[D\left(A^{n-1}\right)\right]$. Hence the equality holds for $x \in D\left(A^{n-1}\right)$.

Proof of $(\mathrm{f}) \rightarrow($ b). We need the following lemma. The straightforward proof is omitted.

LEMMA 4.7. Let $\mu_{0}$ be in the resolvent set of a linear operator $A$. If

$$
S=\sum_{k=0}^{\infty}\left(\mu-\mu_{0}\right)^{k} \frac{1}{k !}\left(\mu_{0}^{1-n} R\left(\mu_{0}, A\right)\right)^{(k)}
$$

exists in $L(E)$, then $\mu$ is in the resolvent set of $A$ and $R(\mu, A)=\mu^{n-1} S$.

We show first that we obtain from the growth estimates on the derivatives of the resolvent for real $\mu>w^{\prime}$ the estimate

$$
|R(\mu, A) x| \leq M|\mu|^{n-1}\left(\frac{1}{\operatorname{Re} \mu-w}\right)|x|
$$

for all $x \in E$ and all complex $\mu$ with $\operatorname{Re} \mu>w=\max \left\{w^{\prime}, 0\right\}$.

Let $\mu \in C$ with $\operatorname{Re} \mu>w$. Choose $r>w$ such that $|\mu-r|<$ $r-w$. Then the operator $S$, as defined in Lemma 4.7 exists and satisfies $|S| \leq M[r-w-|\mu-r|]^{-1}$. Hence, for $r \rightarrow \infty$ we obtain, by Lemma 4.7, the inequality (*). By the resolvent identity (4.2) and the inequality (*) we obtain $|R(\mu, A) x| \leq M|x|_{n-1}$ for every $x \in D\left(A^{n-1}\right)$ and $\operatorname{Re} \mu$ sufficiently large. Now let $x \in D\left(A^{n+2}\right) \subset D\left(A^{3}\right)$. Then 
$A^{3} x \in D\left(A^{n-1}\right)$ and $\left|R(\mu, A) A^{3} x\right| \leq M(x)$. By Proposition 2.2, there is a solution $u(\cdot)$ of (ACP) with initial value $x$ and $|u(t)| \leq C e^{p t}$. By (2.2) and (*), the solution is unique. By Lemma 4.6 and by partial integration we obtain for sufficiently large $\operatorname{Re} \mu$ that

$$
R(\mu, A) x=\int_{0}^{\infty} e^{-\mu t} u(t) d t=\mu^{n-1} \int_{0}^{\infty} e^{-\mu t} f(t) d t
$$

where $f(t)$ is the $(n-1)$-times integrated solution $u(t)$. Hence

$$
\mu^{1-n} R(\mu, A) x=\int_{0}^{\infty} e^{-\mu t} f(t) d t .
$$

For $x^{*} \in E^{*}$ we define

$$
f(\mu):=\left[\mu^{1-n} R(\mu, A) x, x^{*}\right]=\int_{0}^{\infty} e^{-\mu t}\left[f(t), x^{*}\right] d t .
$$

Using the Post-Widder inversion formula of the Laplace transform (see, for example, [28], p. 35) and the growth condition of (f) we obtain

$$
\begin{aligned}
\left|\left[f(t), x^{*}\right]\right| & =\left|\lim _{k \rightarrow \infty}(-1)^{k} \frac{1}{k !}\left(\frac{k}{t}\right)^{k+1} F^{(k)}\left(\frac{k}{t}\right)\right| \\
& \leq M \lim _{k \rightarrow \infty}\left(\frac{k / t}{k / t-w}\right)^{k+1}|x|\left|x^{*}\right|=M e^{w t}|x|\left|x^{*}\right| .
\end{aligned}
$$

By the uniform boundedness principle, we get for every $x$ in the dense set $D\left(A^{n+2}\right)$ a solution $u(\cdot)$ of (ACP) such that the $(n-1)$-times integrated solution $f(t)$ satisfies $|f(t)| \leq M e^{w t}|x|=M e^{w t}|u(0)|$.

Proof of $(\mathrm{e}) \rightarrow(\mathrm{b})$. If (e) holds for $A$, then there exists a $\mu_{0}$ and a $w \geq 1$, such that 0 is in the resolvent set of $A_{0}=\mu_{0}+A$ and $\left|R\left(\mu, A_{0}\right)^{k} x\right| \leq M(\mu-w)^{-k}|x|_{n-1}$ for all $k \in N, \mu>w \geq 1$ and $x \in D\left(A_{0}^{n-1}\right)$. From the resolvent identity

$$
\mu^{1-n} R(\mu, A) A^{n-1} x=R(\mu, A) x-\sum_{k=0}^{n-1} \mu^{-(k+1)} A^{k} x,
$$

it follows by induction that

$$
\begin{aligned}
& (-1)^{k}(1 / k !)\left(d^{k} / d \mu^{k}\right)\left[\mu^{1-n} R(\mu, A) A^{n-1} x\right] \\
& \quad=R(\mu, A)^{k+1} x-\sum_{j=0}^{n-1}\left(\begin{array}{c}
j+k \\
k
\end{array}\right) \mu^{-(j+k+1)} A^{j} x .
\end{aligned}
$$


The operator $A_{0}$ is invertible; therefore

$$
\begin{aligned}
& (-1)^{k}(1 / k !)\left(d^{k} / d \mu^{k}\right)\left[\mu^{1-n} R\left(\mu, A_{0}\right) z\right] \\
& \quad=R\left(\mu, A_{0}\right)^{k+1} A_{0}^{1-n} z-\sum_{j=0}^{n-1}\left(\begin{array}{c}
j+k \\
k
\end{array}\right) \mu^{-(j+k+1)} A_{0}^{j+1-n} z
\end{aligned}
$$

and hence

$$
\begin{aligned}
& \left|(1 / k !)\left(d^{k} / d \mu^{k}\right)\left[\mu^{1-n} R\left(\mu, A_{0}\right) z\right]\right| \\
& \quad \leq M(\mu-w)^{-(k+1)}\left|A_{0}^{1-n} z\right|_{n-1}+\sum_{j=0}^{n-1}\left(\begin{array}{c}
j+k \\
k
\end{array}\right) \mu^{-(j+k+1)}\left|A_{0}^{j+1-n} z\right| \\
& \quad \leq C\left[(\mu-w)^{-(k+1)}+\frac{1}{\mu^{k+1}} \sum_{j=0}^{n-1}\left(\begin{array}{c}
j+k \\
k
\end{array}\right) \mu^{-j}\right]|z|
\end{aligned}
$$

for $z \in E$ and $\mu>w \geq 1$. By the generalized Bernoulli Inequality

$$
\sum_{j=0}^{n-1}\left(\begin{array}{c}
j+k \\
k
\end{array}\right) x^{j} \leq\left(1+x+\cdots+x^{n-1}\right)^{k+1}
$$

which can be proved by induction, we obtain

$$
\begin{aligned}
& \left|(1 / k !)\left(d^{k} / d \mu^{k}\right)\left[\mu^{1-n} R\left(\mu, A_{0}\right) z\right]\right| \\
& \quad \leq C\left[(\mu-w)^{-(k+1)}+\left(1 / \mu^{k+1}\right)\left(1+1 / \mu+\cdots+(1 / \mu)^{n-1}\right)^{k+1}\right]|z| \\
& \quad \leq C\left[(\mu-w)^{-(k+1)}+(\mu-1)^{k+1}\right]|z| \leq 2 C(\mu-w)^{-(k+1)}|z|
\end{aligned}
$$

for all $\mu>w \geq 1$ and $z \in E$. Therefore, (f) $\rightarrow$ (b) holds for the operator $A_{0}$. But if (b) holds for $A_{0}$, then (b) holds also for $A$.

In the following theorem, due to Sova [30] and Lions [20], we see that the union of all classes $G_{n}$ of densely defined generators of $n$-times integrated semigroups coincides with the class of generators of exponentially bounded distribution semigroups. However, the approach to exponentially wellposed Cauchy problems via integrated semigroups is technically easier and gives sharper results because the parameter $n$ is specified.

TheOREM 4.8. Let $A$ be a densely defined linear operator on a Banach space $E$. Then the following statements are equivalent.

(a) A generates an integrated semigroup. 
(b) There exist real constants $M, w$ and $k \in N_{0}$ such that $R(\mu, A)$ exists and satisfies $|R(\mu, A)| \leq M(1+|\mu|)^{k}$ for all $\mu \in C$ with $\operatorname{Re} \mu>w$.

(c) A generates an exponentially bounded semigroup of distributions.

Proof. The equivalence of (b) and (c) was proved by Lions [20]. The implication (a) $\rightarrow$ (b) follows from Theorem 4.5, (f) and the implication (b) $\rightarrow$ (a) follows from Proposition 2.3 and Theorem 4.5 (a).

In applications it is often easy to check condition (b) of the previous theorem. From Proposition 2.3 and Theorem 4.5 (a) we obtain the following corollary.

Corollary 4.9. Let $A$ be a linear operator on a Banach space $E$. If there are constants $M, w, k$ such that $R(\mu, A)$ exists and satisfies $|R(\mu, A)| \leq M(1+|\mu|)^{k}$ for all $\mu \in C$ with $\operatorname{Re} \mu>w$, then (ACP) is (at least) exponentially $(k+3, k+3)$-wellposed. If, in addition, $A$ is densely defined, then $A$ generates (at least) $a(k+3)$-times integrated semigroup.

If the norm estimate $|R(\mu, A)| \leq M(1+|\mu|)^{k}$ holds for all $M$ in the angular region $\Sigma_{\alpha}=\{\mu:|\arg \mu| \leq \pi / 2+\alpha\}$ for some $0<\alpha<\pi / 2$ then, as shown in [19], for every $x \in E$ the inverse Laplace transform (ILP) is a $C^{\infty}$-function which solves $u^{\prime}(t)=A u(t)$ for $t>0$ (not necessarily for $t \geq 0$ ). Moreover, if $\lim _{t \rightarrow 0} u(t)=x$ exists (by the previous corollary, the limit exists at least for all $\left.x \in D\left(A^{k+3}\right)\right)$, then $|u(t)| \leq M t^{-k}|x|, 0<t \leq 1$, for a constant $M$ not depending on $x$.

In the following corollary we prove a theorem due to Arendt [1]. Similar results can be found in [22].

COROLlaRy 4.10. Let $A$ be a densely defined operator on a Banach space $E$, ordered by a normal and generating cone. If there exists $a w \in R$ such that every $\mu>w$ is in the resolvent set of $A$ and if $R(\mu, A) x \geq 0$ for all $x \geq 0$ and $\mu>w$, then (ACP) is exponentially $(2,1)$-wellposed.

Proof. Without loss of generality we assume that $w<0$. By

$$
R(0, A)=R(\mu, A)+\cdots+\mu^{n-1} R(\mu, A)^{n}+\mu^{n} R(\mu, A)^{n} R(0, A),
$$

by the positivity of $R(\mu, A)$ for $\mu>0$ and by the assumption that the cone is generating and normal, we obtain $\left|\mu^{n} R(\mu, A)^{n} R(0, A)\right| \leq M$ and hence, for $x \in D(A),\left|R(\mu, A)^{n} x\right| \leq M \mu^{-n}|x|_{1}$ for all $n \in N$ 
and $\mu>0$. Therefore, by Theorem 4.5 (e), (ACP) is exponentially $(2,1)$-wellposed.

If we reformulate the growth conditions of Theorem 4.5 (f) on the derivatives of the function $\mu \rightarrow 1 / \mu^{1-n} R(\mu, A)$ into growth conditions on powers of the resolvents, one may get an idea why statement (f) of Theorem 4.5 is only theoretically important. The proof of the lemma is omitted.

LEMMA 4.11. Let $A$ be a linear operator with nonempty resolvent set. Then, for $n \geq 2$,

$$
\begin{aligned}
& \left(\mu^{1-n} R(\mu) x\right)^{(k)} \\
& \quad=(-1)^{k} \frac{k !}{(n-2) !} \mu^{1-n-k} \sum_{j=0}^{k} \frac{(n+k-j-2) !}{(k-j) !} \mu^{j} R(\mu, A)^{j+1} x .
\end{aligned}
$$

Related results. For densely defined operators $A$, Davies and Pang [4] developed the theory of "exponentially bounded $C$-semigroups". If one sets $R=R(\mu, A)^{n-1}$, then the statement of their main theorem is exactly the implication (e) $\rightarrow$ (a) of Theorem 4.5.

THEOREM ([4]). If $A$ is a densely defined, closed operator on a Banach space $E$ such that there exists an injective bounded operator $R$ with dense range satisfying.

(i) $(\mu-A)$ is injective for all $\mu>a$,

(ii) Range $(R) \subset D\left((\mu-A)^{-n}\right)$ for all $\mu>a$ and $n \in N_{0}$,

(iii) $\left|(\mu-A)^{-n} R\right| \leq M(\mu-a)^{-n}$ for all $\mu>a$ and $n \in N_{0}$,

(iv) $(\mu-A)^{-1} R x=R(\mu-A)^{-1} x$ for all $\mu>a$ and $f \in \operatorname{Range}(\mu-A)$, then (ACP) has unique solutions for all $x \in R D(A)$. The solutions depend continuously on the initial value $x$ given in the $R^{-1}$-graph norm on $R D(A)$.

Recently, Arendt [2] has obtained the following generalization of Widder's classical characterization of those real-valued functions which are the Laplace transform of a bounded real function.

THEOREM ([2]). Let $a \geq 0$ and let $R(\cdot)$ be a function from $(a, \infty)$ into a Banach space $E$. The following statements are equivalent:

(i) $R(\cdot) \in C^{\infty}((a, \infty), E)$ and there exist a constant $M \geq 0$ and $w \in(-\infty, a]$ such that for all $k \in N$ and all $\mu>a$ :

$$
\left|\frac{1}{n !} R(\mu)^{(k)}\right| \leq M\left(\frac{1}{\mu-w}\right)^{n+1} \text {. }
$$


(ii) There exists a function $F(\cdot):[a, \infty) \rightarrow E$ satisfying $F(0)=0$ and $|F(t+h)-F(t)| \leq M h e^{w(t+h)}(t \geq 0, h \geq 0)$ such that $R(\mu)=$ $\mu \int_{0}^{\infty} e^{-\mu t} F(t) d t$ for all $\mu>a$.

If $A$ is a nondensely defined linear operator such that the interval $(a, \infty)$ is contained in the resolvent set of $A$ and if one defines $R(\mu):=$ $\mu^{1-n} R(\mu, A)$, then the theorem above yields immediately the following corollary which extends Sova's characterization of densely defined generators to nondensely defined generators of integrated semigroups.

COROLlary ([2]). Let $A$ be a nondensely defined linear operator on a Banach space $E$. If statement (f) of Theorem 4.8 is fulfilled, then $A$ generates an n-times integrated semigroup.

5. Integrated semigroups are integrated, strongly continuous semigroups. If $A$ generates a strongly continuous semigroup, then, as mentioned above, $A$ generates an $(n-1)$-times integrated semigroup on $E$ for all $n \in N$. If $A$ generates an $(n-1)$-times integrated semigroup $(S(t))$ on $E$, then, assuming that the name "integrated semigroup" makes sense, one would expect that the $(n-1)$ th derivatives of $(S(t))$ define a strongly continuous semigroup on an appropriate Banach space $F$. This will be shown in the following. First we collect some basic properties of integrated semigroups.

LEMMA 5.1. Let $A$ be the generator of an $(n-1)$-times integrated semigroup $(S(t))$. Then, if $n=1,(S(t))$ is a strongly continuous semigroup. If $n \geq 2$, then

(a) $S(t) x=0$ for all $t \geq 0$ implies $x=0$, and $S(0)=0$.

(b) For every $x \in D(A)$ we have $S(t) x \in D(A), A S(t) x=S(t) A x$ and

$$
S(t) x=\int_{0}^{t} S(s) A x d s+\frac{1}{(n-1) !} t^{n-1} x .
$$

(c) For every $x \in E$ we have $\int_{0}^{t} S(s) x d s \in D(A)$ and

$$
A \int_{0}^{t} S(s) x d s=S(t) x-t^{n-1} x /(n-1) ! .
$$

Hence, for every $x \in E$, the function $t \rightarrow S(t) x$ is a solution of the $n$-times integrated (ACP) or a "mild" solution of (ACP).

(d) For every $x \in D\left(A^{n}\right)$ the unique solution of (ACP) is given by (4.1). If $x(\cdot)$ is a solution of (ACP), then

$$
S(t) x(0)=\int_{0}^{t} \frac{1}{(n-2) !}(t-s)^{n-2} x(s) d s .
$$


(e)

$$
\begin{aligned}
S(t) S(s) x=\int_{0}^{t} \frac{1}{(n-2) !}\left[(t-r)^{n-2} S(r+s) x\right. & \\
& \left.-(t+s-r)^{n-2} S(r) x\right] d r
\end{aligned}
$$

for every $x \in E$ and $t, s \geq 0$.

Proof. The statement (a) is obvious. Statements (b) and (d) were shown in Lemmas 4.3 and 4.4 and in (4.1). Statement (c) follows from

$$
\int_{0}^{t} S(s) x d s=\mu R(\mu, A) \int_{0}^{t} S(s) x d s-\int_{0}^{t} S(s) A R(\mu, A) x d s .
$$

Using statement (c) one obtains

$$
\begin{aligned}
\int_{0}^{t} S(s) x d s= & \mu R(\mu, A) \int_{0}^{t} S(s) x d s \\
& -\left(S(t)+\frac{1}{(n-1) !} t^{n-1} I\right) R(\mu, A) x
\end{aligned}
$$

Since $A$ commutes with $S(t)$ on $D(A)$, the resolvent commutes with $S(t)$ on $E$. Therefore, for every $x \in E$, we have $\int_{0}^{t} S(s) x d s \in D(A)$ and

$$
(\mu-A) \int_{0}^{t} S(s) x d s=\mu \int_{0}^{t} S(s) x d s-S(t) x-t^{n-1} x /(n-1) !
$$

To prove statement (f) let $x \in D\left(A^{n}\right)$ and let $x(\cdot)$ be the solution of $(\mathrm{ACP})$ with $x(0)=x$. Let

$$
x^{[j]}(t)=\int_{0}^{t} \frac{1}{(j-1) !}(t-s)^{j-1} x(s) d s
$$

be the $j$-times integrated solution $x(\cdot)$. Define, for $s>0$,

$$
\begin{aligned}
u(t) & =S(t+s) x-S(t) x-\sum_{k=1}^{n-2} \frac{1}{k !} s^{k} \int_{0}^{t} \frac{1}{(n-2-k) !}(t-r)^{n-2-k} x(r) d r \\
& =x^{[n-1]}(t+s)-x^{[n-1]}(t)-\sum_{k=1}^{n-2} \frac{1}{k !} s^{k} x^{[n-1-k]}(t) .
\end{aligned}
$$

Then $u(0)=S(s) x, u(t) \in D(A)$ for all $t \geq 0$ and by $d / d t x^{[j]}(t)=$ $x^{[j-1]}(t)$ and

$$
A x^{[j]}(t)=x^{[j-1]}(t)-\frac{1}{(j-1) !} t^{j-1} x
$$


we obtain $A u(t)-u^{\prime}(t)=0$. Hence $u(\cdot)$ is a solution of (ACP) with $u(0)=S(s) x$. Therefore

$$
\begin{aligned}
S(t) S(s) x= & \int_{0}^{t} \frac{1}{(n-2) !}(t-r)^{n-2}(S(r+s) x-S(r) x) d r \\
& -\sum_{k=1}^{n-2} \frac{1}{k !} s^{k} \int_{0}^{t} \frac{1}{(n-2-k) !}(t-r)^{n-2-k} S(r) x d r \\
= & \int_{s}^{s+t} \frac{1}{(n-2) !}(t+s-r)^{n-2} S(r) x d r \\
& -\sum_{k=0}^{n-2} \frac{1}{k !} s^{k} \int_{0}^{t} \frac{1}{(n-2-k) !}(t-r)^{n-2-k} S(r) x d r \\
= & \int_{s}^{s+t} \frac{1}{(n-2) !}(t+s-r)^{n-2} S(r) x d r \\
& -\int_{0}^{t} \frac{1}{(n-2) !}(t+s-r)^{n-2} S(r) x d r \\
= & \int_{0}^{t} \frac{1}{(n-2) !}\left[(t-r)^{n-2} S(r+s) x\right. \\
&
\end{aligned}
$$

Kellermann [14] proved that every once integrated semigroup in fact defines a strongly continuous semigroup on an appropriate Banach space $F$. Using Lemma 5.1 we will extend Kellermann's result for arbitrary $(n-1)$-times integrated semigroups.

THEOREM 5.2. Let $A$ be the generator of an $(n-1)$-times integrated semigroup $(S(t))$ with $|S(t)| \leq M e^{w t}$ and let the mth differentiation space of $(S(t))$ be defined by $C^{m}:=\left\{x \in E: t \rightarrow S(t) x \in C^{m}\left(R^{+}, E\right)\right\}$. Then

(a) $\cdots D\left(A^{3 n-3}\right) \subset C^{3 n-3} \subset D\left(A^{2 n-2}\right) \subset C^{2 n-2} \subset D\left(A^{n-1}\right) \subset C^{n-1}$.

(b) $|x|_{F}:=\sup _{t \geq 0}\left|e^{-w t} S^{(n-1)}(t) x\right|$ defines a norm on $D\left(A^{n-1}\right)$ with $|x| \leq|x|_{F} \leq C|x|_{n-1}$.

(c) Let $F$ be the Banach space obtained by the closure of $C^{2 n-2}$ under | $\left.\right|_{F}$; then $\left.\overline{D\left(A^{2 n-2}\right)}\right|_{F}=F \subset C^{n-1}$.

(d) If $A$ is densely defined, then ${\overline{D\left(A^{n-1}\right)}}^{||_{F}}=F \subset C^{n-1}$.

(e) The restriction $A_{/ F}$ of $A$ to $F$ with domain $D\left(A_{/ F}\right):=\{x \in$ $D(A) \cap F: A x \in F\}$ and $A_{/ F} x=A x$ for all $x \in D\left(A_{/ F}\right)$ is the generator of the strongly continuous semigroup $(T(t)):=\left(S^{(n-1)}(t)_{/ F}\right) \subset L(F)$ satisfying $|T(t)|_{L(F)} \leq e^{w t}$. 
Proof. From the proof of Lemma 5.1 (e) we obtain $\begin{aligned}(\mathrm{L} .0) S(t) S(s) x= & \int_{0}^{t} \frac{1}{(n-2) !}(t-r)^{n-2}(S(r+s) x-S(r) x) d r \\ & -\sum_{k=1}^{n-2} \frac{1}{k !} s^{k} \int_{0}^{t} \frac{1}{(n-2-k) !}(t-r)^{n-2-k} S(r) x d r\end{aligned}$

and

$$
S(s) C^{m} \subset C^{m+1} \text { for all } m \geq 0 .
$$

Using (L.0) one shows by induction that for all $1 \leq k \leq n-2$ :

$$
\begin{aligned}
S(t) S^{(k)}(s)= & \frac{1}{(n-2-k) !} \int_{0}^{t}(t-r)^{n-2-k} S(r+s) d r \\
& -\sum_{j=0}^{k-1} \frac{1}{(n-2-j) !} t^{n-2-j} S^{(k-1-j)}(s) \\
& -\sum_{j=k}^{n-2} \frac{1}{(j-k) !} s^{j-k} \\
& \times \int_{0}^{t} \frac{1}{(n-2-j) !}(t-r)^{n-2-j} S(r) d r
\end{aligned}
$$

From (L.1) and (L.2) we conclude that $S^{(k)}(s) C^{m} \subset C^{m+1}$ for $0 \leq k \leq$ $n-2$ and $m \geq 0$. Using (L.2), an induction shows that $S(t) S^{(k)}(0)=0$ for all $1 \leq k \leq n-2$. By Lemma 5.1 (a) we obtain

(L.3) $S^{(k)}(s) C^{m} \subset C^{m+1}$ for $0 \leq k \leq n-2$ and $m \geq 0$,

$$
S^{(k)}(0)=0 \text { for all } 0 \leq k \leq n-2 \text {. }
$$

Differentiating (L.2) for $k=n-2$ we get

$$
\begin{array}{r}
S(t) S^{(n-1)}(s)=S(t+s)-\sum_{j=0}^{n-2} t^{j} / j ! S^{(j)}(s) \text { and } \\
S^{(n-1)}(s) C^{m} \subset C^{m} \text { for all } m \geq n-1 .
\end{array}
$$

Moreover, it follows from (L.4) and (L.3) that

$$
S(t)\left[S^{(n-1)}(0)-I_{/ C^{n-1}}\right]=0 .
$$

Hence, by Lemma 5.1 (a),

$$
S^{(n-1)}(0)=I \text { on } C^{n-1} \text {. }
$$


From (L.4) and (L.5) we conclude that $S^{(n-1)}(t) S^{(n-1)}(s)=$ $S^{(n-1)}(t+s)$ on $C^{m}$ for all $m \geq n-1$. This and (L.6) yield

(L.7) $S^{(n-1)}(t)$ defines a strongly continuous semigroup on $C^{m}$ for all $m \geq n-1$.

From Lemma 5.1 we know that $S(t)$ commutes with $A$ on $D(A)$. Hence $S(t)$ commutes with $R(\mu, A)$ on $E$ and we have

$$
S^{(k)}(t) R(\mu, A)=R(\mu, A) S^{(k)}(t) \text { on } C^{k} \text { for } k \geq 0 .
$$

Differentiating (L.4) we obtain

$$
\begin{gathered}
S(t) S^{(n)}(s)=S^{(1)}(t+s)-\sum_{j=0}^{n-2} t^{j} / j ! S^{(j+1)}(s) \text { and } \\
S^{(n)}(s) C^{m} \subset C^{m-1} \text { for all } m \geq n .
\end{gathered}
$$

From (L.9) it follows that $S(t) S^{(n)}(0)=S^{(1)}(t)-t^{n-2} /(n-1) ! I$ on $C^{m}$ for $m \geq n$. But then

$$
\begin{aligned}
R(\mu, A) S^{(n)}(0)= & \mu^{n-1} \int_{0}^{\infty} e^{-\mu t} S^{(1)}(t) d t \\
& -\mu^{n-1} \int_{0}^{\infty} e^{-\mu t} t^{n-2} /(n-2) ! d t=\mu R(\mu, A)-I
\end{aligned}
$$

for $m \geq n$. Hence

$$
R(\mu, A)\left(I-S^{(n)}(0)\right)=I \text { on } C^{m} \text { for all } m \geq n .
$$

Let $x \in C^{m}$ for some $m \geq n$. Then, by (L.10),

$$
x=R(\mu, A)\left(x-S^{(n)}(0) x\right)=R(\mu, A) z \quad \text { for } z \in C^{m-1} .
$$

Hence $C^{m} \subset R(\mu, A) C^{m-1}$ for all $m \geq n$. By

$$
S(s) R(\mu, A)=\mu^{n-1} \int_{0}^{\infty} e^{-\mu t} S(s) S(t) x d t
$$

and (L.1) one shows that $R(\mu, A) C^{m} \subset C^{m+1}$ for all $m \geq 0$. Therefore,

$$
R(\mu, A) C^{m-1}=C^{m} \text { for all } m \geq n .
$$

The function $S(t) x$ is $m$-times continuously differentiable for all $x \in$ $D\left(A^{m}\right)$ (Lemma $5.1(\mathrm{~b})$ ). Hence $D\left(A^{m}\right) \subset C^{m}$ for all $m \geq 0$. This and (L.12) gives

$$
D\left(A^{n}\right) \subset C^{n}=R(\mu, A) C^{n-1} \subset R(\mu, A) E=D(A) .
$$


Hence $C^{m} \subset D(A)$ for all $m \geq n$. Therefore, by (L.11),

$$
S^{(n)}(0) x=A x \quad \text { for all } x \in C^{m} \text { and all } m \geq n \text {. }
$$

Combining (L.14) and (L.12) and (L.10) we obtain

(L.15) $A C^{m} \subset C^{m-1}$ and $(\mu-A) C^{m}=C^{m-1}$

for $m \geq n$ and $\mu \in \rho(A)$.

From (L.13) we get $R(\mu, A) D\left(A^{n}\right) \subset R(\mu, A) C^{n} \subset R(\mu, A) D(A)$. by (L.12), $D\left(A^{n+1}\right) \subset C^{n+1} \subset D\left(A^{2}\right)$. Repeating this procedure we get

$$
\cdots C^{3 n-3} \subset D\left(A^{2 n-2}\right) \subset C^{2 n-2} \subset D\left(A^{n-1}\right) \subset C^{n-1} .
$$

Now let $x \in C^{2 n-2}$. Then, by (L.3), $S^{(n-2)}(h) x / h \in C^{2 n-2}$ for all $h>0$. Moreover, by (L.15), $A^{j} x \in C^{n-1}$ for $0 \leq j \leq n-1$. Hence, by $S^{(n-1)}(0)=I$ on $C^{n-1}$ (by (L.6)) we get $\left|A^{j} x-(1 / h) S^{(n-2)}(h) A^{j} x\right| \rightarrow 0$ as $h \rightarrow 0$ for all $0 \leq j \leq n-1$. By $\left|x-(1 / h) S^{(n-2)}(h) x\right|_{n-1} \rightarrow 0$ as $h \rightarrow 0$ we get that $C^{2 n-1}$ is dense in $C^{2 n-2}$ with respect to ||$_{n-1}$. The same argument shows that $C^{2 n}$ is dense in $C^{2 n-1}$ with respect to | $\left.\right|_{n-1}$. Therefore $C^{2 n}$ is dense in $C^{2 n-2}$ with respect to ||$_{n-1}$. Repeating this procedure we obtain

(L.17) $C^{m}$ is dense in $C^{2 n-2}$ with respect to ||$_{n-1}$ for $m \geq 2 n-2$.

Especially, by (L.16),

$$
D\left(A^{2 n-2}\right) \text { is dense in } C^{2 n-2} \text { with respect to }||_{n-1} \text {. }
$$

For every $x \in D\left(A^{n-1}\right), t \rightarrow S(t) x$ is $(n-1)$-times continuously differentiable (Lemma 5.1 (b)) and

(L.19) $S^{(n-1)}(t) x=S(t) A^{n-1} x+\sum_{k=0}^{n-2} \frac{1}{k !} t^{k} A^{k} x \quad$ for $x \in D\left(A^{n-1}\right)$.

Hence, for all $x \in D\left(A^{n-1}\right),\left|S^{(n-1)}(t) x\right| \leq C e^{w t}|x|_{n-1}$. We define a norm on $D\left(A^{n-1}\right)$ by $|x|_{F}=\sup _{t \geq 0}\left|e^{-w t} S^{(n-1)}(t) x\right|$. From $S^{(n-1)}(0) x$ $=x$ we get

$$
|x| \leq|x|_{F} \leq C|x|_{n-1} .
$$

Let $F$ be the closure of $C^{2 n-2}$ with respect to this norm. Then $F$ is a Banach space. Let $x \in F$ and let $x_{n} \rightarrow x$ with respect to the $F$-norm. By (L.20), $x_{n} \rightarrow x$ in $E$. Hence $S(t) x_{n}$ converges to $S(t) x$ in $E$ uniformly on compacts. The functions $S(\cdot) x_{n}$ are $(n-1)$-times 
continuously differentiable. From (L.7) we know that $S^{(n-1)}(t)$ defines a semigroup on $C^{2 n-2}$. Hence, for $x \in C^{2 n-2}$,

$$
\left|S^{(n-1)}(s) x\right|_{F}=\sup _{t \geq 0}\left|e^{-w t} S^{(n-1)}(t+s) x\right| \leq e^{w s}|x|_{F}
$$

By

$$
\left|S^{(n-1)}(t) x_{n}-S^{(n-1)}(t) x_{m}\right| \leq e^{w s}\left|x_{n}-x_{m}\right|_{F} \quad \text { and } \quad S^{(k)}(0) x_{n}=0
$$

we conclude that $t \rightarrow S(t) x$ is $(n-1)$-times continuously differentiable. Therefore $x \in C^{n-1}$ and

$$
F \subset C^{n-1} \text {. }
$$

Now let $x \in F \subset C^{n-1}$. Then $S^{(n-1)}(t) x$ is well-defined. If $x_{n}$ is a sequence in $C^{2 n-2}$ converging to $x$ with respect to the $F$-norm, then, $S^{(n-1)}(t) x_{n} \in C^{2 n-2}$ is a Cauchy sequence in $F$. Therefore, $S^{(n-1)}(t) x_{n}$ converges to $g \in F$ with respect to the $F$-Norm and, by (L.20), also in $E$. Therefore, $g=S^{(n-1)}(t) x \in F$. This and (L.7) shows that $S^{(n-1)}(t)_{/ F}$ is a semigroup on $F$. We have to show the strong continuity. From (L.18) we know that $D\left(A^{2 n-2}\right)$ is dense in $C^{2 n-2}$ with respect to | $\left.\right|_{n-1}$. But then, by (L.20), $D\left(A^{2 n-2}\right)$ is dense in $C^{2 n-2}$ with respect to the $F$-norm. Therefore we have

$F$ is the $F$-closure of $D\left(A^{2 n-2}\right)$.

Now let $x \in D\left(A^{2 n-2}\right)$. Then, by (L.19),

$$
\begin{aligned}
\left|S^{(n-1)}(t) x-x\right|_{F} & =\left|S(t) A^{n-1} x+\sum_{k=1}^{n-2} \frac{1}{k !} t^{k} A^{k} x\right|_{F} \\
& \leq\left|S(t) A^{n-1} x+\sum_{k=1}^{n-2} \frac{1}{k !} t^{k} A^{k} x\right|_{n-1} \rightarrow 0 \quad \text { as } t \rightarrow 0 .
\end{aligned}
$$

From (L.21) we get $\left|S^{(n-1)}(s) x\right|_{F} \leq e^{w s}|x|_{F}$ for $x \in F$. Therefore, by (L.23), $\left|S^{(n-1)}(t) x-x\right|_{F} \rightarrow 0$ as $t \rightarrow 0$ for all $x \in F$.

Hence $T(t):=S^{(n-1)}(t)_{/ F}$ is a strongly continuous semigroup on $F$ with $|T(t)|_{L(F)} \leq e^{w t}$. Let $B$ be the generator of $T(t)$. If $T(\cdot) x$ is $F$ differentiable at 0 , then, by $($ L.20), $T(\cdot) x$ is $E$-differentiable. Therefore $D(B) \subset C^{n}$. By (L.13) and (L.14) we get $D(B) \subset C^{n} \subset D(A)$ and $B x=A x$ for all $x \in D(B)$.

Let $A_{/ F}$ be the restriction of $A$ to $F$. Then $D(B) \subset D\left(A_{/ F}\right)$ and $B x=A_{/ F} x$ for all $x \in D(B) . A_{/ F}$ is a closed operator on $F$. Moreover, for every $\mu \in \rho(A) \cap \rho(B),\left(\mu-A_{/ F}\right)$ is one-to-one. From $\left(\mu-A_{/ F}\right) R(\mu, B) x=(\mu-B) R(\mu, B) x=x$ for all $x \in F$ we get that 
$\left(\mu-A_{/ F}\right)$ is onto. Hence $R\left(\mu, A_{/ F}\right)$ exists and $R\left(\mu, A_{/ F}\right)=R(\mu, B)$. From this we get $D(B)=D\left(A_{/ F}\right)$.

From (L.22) and (L.23) we obtain $\left.\overline{D\left(A^{2 n-2}\right)}\right|^{\left.\right|_{F}}=F \subset C^{n-1}$. If $A$ is densely defined, then, by Lemma $2.1, D\left(A^{2 n-2}\right)$ is ||$_{n-1}$-dense and therefore also ||$_{F}$-dense in $D\left(A^{n-1}\right)$. But then $\left.\overline{D\left(A^{n-1}\right)}\right|_{F}=$ $F \subset C^{n-1}$. This finishes the proof of Theorem 5.2.

Corollary 5.3. Let $A$ be a densely defined operator on a Banach space $E$. Then the following statements are equivalent.

(i) $A$ is the generator of an $(n-1)$-times integrated semigroup.

(ii) The resolvent set of $A$ is nonempty and there exists a norm $|\quad| F$ on $D\left(A^{n-1}\right)$ such that $|x| \leq|x|_{F} \leq C|x|_{n-1}$. The restriction of $A$ to $F=\left.\overline{D\left(A^{n-1}\right)}\right|^{\mid}$generates a strongly continuous semigroup on $F$.

Proof. The implication (i) $\rightarrow$ (ii) is contained in Theorem 5.2. If (ii) holds, then, for every initial value $x \in D\left(A_{/ F}\right)$, there exists a unique solution $u(\cdot): R^{+} \rightarrow D\left(A_{/ F}\right)$ in $C^{1}\left(R^{+}, F\right)$ of the Cauchy problem for $A_{/ F}$ satisfying $|u(t)|_{F} \leq M e^{w t}|x|_{F} . D\left(A_{/ F}\right)$ is ||$_{F}$-dense and hence $\mid$ |-dense in $F$ and $F$ is | |-dense in $E$ (by Lemma 2.1). Therefore, $D:=D\left(A_{/ F}\right)$ is dense in $E$ and, for every initial value $x \in D$, there exists a unique solution $u(\cdot): R^{+} \rightarrow D\left(A_{/ F}\right) \subset D(A)$ in $C^{1}\left(R^{+}, F\right) \subset$ $C^{1}\left(R^{+}, E\right)$ of the Cauchy problem for $A$ satisfying $|u(t)| \leq|u(t)|_{F} \leq$ $M e^{w t}|x|_{F} \leq M e^{w t}|x|_{n-1}$.

Let $\mu$ be in the resolvent set of $A$ and let $x \in D$. Then the function $w(t)=R(\mu, A) u(t)$ is a solution of (ACP) with $|w(t)| \leq M^{\prime} e^{w t}|x|_{n-2}$. Let $v(\cdot)$ be the once intgrated solution. Then $v(t)=\mu \int_{0}^{t} w(s) d s-$ $w(t)+R(\mu, A) x$. Hence $|v(t)| \leq C e^{c t}|x|_{n-2}$. By induction we obtain that the $(n-1)$-times integrated solution $f(\cdot)$ satisfies $|f(t)| \leq C^{\prime} e^{d t}|x|$. Hence, by Theorem $4.6, A$ is the generator of an $(n-1)$-times integrated semigroup on $E$.

REMARK. Combining Theorem 4.8 and Corollary 5.3 we see that a densely defined operator $A$ on a Banach space $E$ with nonempty resolvent set generates an exponentially bounded distribution semigroup on $E$ if and only if the restriction of $A$ to the Banach space $F$ constructed above generates a strongly continuous semigroup on $F$.

6. Adjoint Cauchy problems. If $\boldsymbol{A}$ is densely defined and closed on a reflexive Banach space, then the adjoint $A^{*}$ is also densely defined (see [9], p. 13). From $R(\mu, A)^{*}=R\left(\mu, A^{*}\right)$ and Theorem 4.5 (f) we get that, on a reflexive Banach space $E$, a densely defined operator $A$ 
generates an (n-1)-times integrated semigroup on $E$ if and only if the adjoint $A^{*}$ generates an $(n-1)$-times integrated semigroup on $E^{*}$.

If $A$ is a densely defined generator of an $j$-times integrated semigroup $(S(t))$ on a nonreflexive Banach space $E$, then the adjoint $A^{*}$ is well-defined (notice that $D\left(A^{* n+1}\right)$ is only weak*-dense in $E^{*}$ (see [13], p. 43)). Define operators $U(t)$ by $U(t) x:=\int_{0}^{t} S(s) x d s$. Then $(U(t))$ is a strongly Lipschitz-continuous operator family in $L(E)$ and, by partial integration, $R(\mu, A) x=\mu^{j+1} \int_{0}^{\infty} e^{-\mu t} U(t) x d t$. The adjoint operator family $\left(U(t)^{*}\right)$ is strongly Lipschitz continuous on $E^{*}$. From $R(\mu, A)^{*}=R\left(\mu, A^{*}\right)$ we conclude that

$$
R\left(\mu, A^{*}\right) x^{*}=\mu^{j+1} \int_{0}^{\infty} e^{-\mu t} U(t)^{*} x^{*} d t
$$

for all $x^{*} \in E^{*}$ and $\mu>w$. Hence $A^{*}$ generates an $(j+1)$-times integrated semigroup.

From the considerations above and Corollary 4.5 we obtain statement (a) and from Theorem 5.2 statement (b) of the following corollary which generalizes Phillips' characterization of the adjoint semigroup (see, for example, [20], p. 38).

COROLlaRY 6.1. Let $A$ be a densely defined generator of an $(n-1)$ times integrated semigroup on a nonreflexive Banach space $E$.

(a) Then $A^{*}$ generates an n-times integrated semigroup $\left(S(t)^{*}\right)$ on $E^{*}$ and the adjoint Cauchy problem $u^{\prime}(t)=A^{*} u(t), u(0)=x^{*}$ is exponentially $(n+1, n)$-wellposed on $E^{*}$.

(b) There exists a norm ||$_{F}$ on $D\left(A^{* n}\right)$ such that $\left|x^{*}\right| \leq\left|x^{*}\right|_{F} \leq$ $C\left|x^{*}\right|_{n}$. Let $C^{2 n}=\left\{x^{*} \in E^{*}: t \rightarrow S(t)^{*} x^{*} \in C^{2 n}\left(R^{+}, E^{*}\right)\right\}$. Then $C^{2 n} \subset D\left(A^{* n}\right)$. Let $F$ be the closure of $C^{2 n}$ with respect to the norm ||$_{F}$. Then the restriction of $A^{*}$ to $F$ generates a strongly continuous semigroup on $F$.

7. The second order Cauchy problem. In this section we study the second order Cauchy problem

$$
u^{\prime \prime}(t)=A u(t) ; \quad u(0)=x, \quad u^{\prime}(0)=y
$$

where $A$ is a not necessarily densely defined linear operator on a Banach space $E$ with nonempty resolvent set (for an investigation of $\left(\mathrm{ACP}_{2}\right)$ for operators with empty resolvent set, see [32]). A function $u(\cdot):[0, \infty) \rightarrow D(A), u(\cdot) \in C^{2}\left(R^{+}, E\right)$ which satisfies $\left(\mathrm{ACP}_{2}\right)$ is called a solution of $\left(\mathrm{ACP}_{2}\right)$. 
In the following we will prove and generalize the classical characterizations of cosine family generators by applying the theorems of the previous sections to the reduced Cauchy problem

$\left(\mathrm{ACP}_{r}\right)$

$$
u^{\prime}(t)=B u(t), \quad u(0)=(x, y)
$$

where $B$ is the operator matrix $\left[\begin{array}{ll}0 & I \\ A & 0\end{array}\right]$ on $E \times E$ (always equipped with the maximum norm) $\left(\mathrm{ACP}_{2}\right)$.

LEMMA 7.1. Let $A$ be a linear operator on a Banach space $E$ with nonempty resolvent set. If $\left(\mathrm{ACP}_{2}\right)$ has unique solutions for all $(x, y) \in$ $D\left(A^{n}\right) \times D\left(A^{m}\right)$, then $\left(\mathrm{ACP}_{2}\right)$ has unique solutions for all $(x, y) \in$ $D\left(A^{n}\right) \times D\left(A^{n}\right)$ and all $(x, y) \in D\left(A^{m+1}\right) \times D\left(A^{m}\right)$.

Proof. $A$ has nonempty resolvent set. Hence $A$ is closed (Lemma 2.1). Without loss of generality we assume that $A^{-1}$ exists. The initial value problem $(*) u^{\prime \prime}(t)=A u(t) ; u(0)=x, u^{\prime}(0)=0$ has unique solutions $u_{x}(\cdot)$ for every $x \in D\left(A^{n}\right)$. The integrated solutions $w_{x}(\cdot)$ solve $u^{\prime \prime}(t)=A u(t) ; u(0)=0, u^{\prime}(0)=x$. Therefore, $\left(\mathrm{ACP}_{2}\right)$ has unique solutions $u_{x}(\cdot)+w_{y}(\cdot)$ for all $(x, y) \in D\left(A^{n}\right) \times D\left(A^{n}\right)$.

For every $x \in D\left(A^{m+1}\right)$, there exists a unique solution $v(t)$ of $u^{\prime \prime}(t)=A u(t) ; u(0)=0, u^{\prime}(0)=A x$. Define $w_{x}(t)=A^{-1} v^{\prime}(t)$. Then $w_{x}(t)$ is a solution of $(*)$ and $\left(\mathrm{ACP}_{2}\right)$ has unique solutions $w_{x}(\cdot)+u_{y}(\cdot)$ for all $(x, y) \in D\left(A^{m+1}\right) \times D\left(A^{m}\right)$.

The straightforward proof of the following lemma is omitted.

LEMMA 7.2. Let $A$ be a linear closed operator on a Banach space $E$. Then $B=\left[\begin{array}{ll}0 & I \\ A & 0\end{array}\right]$ with $D(B)=D(A) \times E$ is a closed operator on the $B$ anach space $E \times E$ equipped with the maximum norm. Moreover,

(a) The resolvent set of $A$ is nonempty if and only if the resolvent set of $B$ is nonempty and, for $\mu \in C$ with $\mu^{2} \in \rho(A)$ $R(\mu, B)=\left[\begin{array}{cc}\mu R\left(\mu^{2}, A\right) & R\left(\mu^{2}, A\right) \\ \mu^{2} R\left(\mu^{2}, A\right)-I & \mu R\left(\mu^{2}, A\right)\end{array}\right]=\left[\begin{array}{cc}\mu R\left(\mu^{2}, A\right) & R\left(\mu^{2}, A\right) \\ A R\left(\mu^{2}, A\right) & \mu R\left(\mu^{2}, A\right)\end{array}\right]$.

(b) $D\left(B^{2 n}\right)=D\left(A^{n}\right) \times D\left(A^{n}\right)$ and, for all $(x, y) \in D\left(B^{2 n}\right)$ :

$$
|x|_{n}+|y|_{n} \leq 2|(x, y)|_{2 n}^{B} \leq 4\left(|x|_{n}+|y|_{n}\right) \text {. }
$$

(c) $D\left(B^{2 n-1}\right)=D\left(A^{n}\right) \times D\left(A^{n-1}\right)$ and, for all $(x, y) \in D\left(B^{2 n-1}\right)$ :

$$
|x|_{n}+|y|_{n-1} \leq 2|(x, y)|_{2 n-1}^{B} \leq 4\left(|x|_{n}+|y|_{n-1}\right) \text {. }
$$

If $\left(\mathrm{ACP}_{2}\right)$ has unique solutions for all $x \in D(A)$ and $y \in E$, then $\left(\mathrm{ACP}_{r}\right)$, has unique solutions for all initial values in $D(B)=D(A) \times E$. 
Moreover, if $A$ has nonempty resolvent set, then, by Lemma 7.2, $B$ has nonempty resolvent set. Hence $B$ is a semigroup generator on $E \times E$, and, by the Phillips Perturbation Theorem (see [11], Theorem 6.4), $C=\left[\begin{array}{ll}0 & 0 \\ A & 0\end{array}\right]$ is a semigroup generator on $E \times E$. But the resolvent set of $C$ is nonempty if and only if $A$ is bounded. Hence $A$ has to be bounded.

Therefore, assuming $A$ having nonempty resolvent set, we will only study Cauchy problems which are uniquely solvable for-at least$(x, y) \in D\left(A^{n}\right) \times D\left(A^{n}\right)=D\left(B^{2 n}\right)$ or for-at least- $(x, y) \in D\left(A^{n+1}\right) \times$ $D\left(A^{n}\right)=D\left(B^{2 n+1}\right)$ where $n \geq 1$.

Definition 7.3. Let $A$ be a linear operator with nonempty resolvent set on a Banach space $E$ and let $n \geq 1$. If there exists a locally bounded function $p(\cdot)$ such that

- $\left(\mathrm{ACP}_{2}\right)$ has unique solutions $u(\cdot)$ for all $(x, y) \in D\left(A^{n}\right) \times D\left(A^{n}\right)$ satisfying $|u(t)| \leq p(t)\left[|x|_{n-1}+|y|_{n-1}\right]$, then $\left(\mathrm{ACP}_{2}\right)$ is called 2n-wellposed.

- $\left(\mathrm{ACP}_{2}\right)$ has unique solutions $u(\cdot)$ for all $(x, y) \in D\left(A^{n+1}\right) \times$ $D\left(A^{n}\right)$ satisfying $|u(t)| \leq p(t)\left[|x|_{n}+|y|_{n-1}\right]$, then $\left(\mathrm{ACP}_{2}\right)$ is called $2 n+1$-wellposed.

If we can choose $p(t)=M e^{w t}$, then $\left(\mathrm{ACP}_{2}\right)$ will be called exponentially $m$-wellposed.

If $u(t)$ is a solution of $\left(\mathrm{ACP}_{2}\right)$, then $w(t)=\left(u(t), u^{\prime}(t)\right)$ is a solution of $\left(\mathbf{A C P}_{r}\right)$. If $w(t)=\left(w_{1}(t), w_{2}(t)\right)$ is a solution of $\left(\mathbf{A C P}_{r}\right)$, then $w(t)=\left(w_{1}(t), w_{1}^{\prime}(t)\right)$ and $w_{1}(t)$ is a solution of $\left(\mathrm{ACP}_{2}\right)$. We have proved the following lemma.

LEMMA 7.4. Let $A$ be a closed linear operator on a Banach space $E$. Then $\left(\mathrm{ACP}_{2}\right)$ has a (unique) solution $u(\cdot)$ if and only if $\left(\mathrm{ACP}_{r}\right)$ has $a$ (unique) solution $\left(u(\cdot), u^{\prime}(\cdot)\right)$.

THEOREM 7.5. Let $A$ be a linear operator with nonempty resolvent set on a Banach space $E$. If $\left(\mathrm{ACP}_{2}\right)$ has unique solutions $u(\cdot)$ for all $(x, y) \in D\left(A^{n}\right) \times D\left(A^{n}\right)\left(\right.$ or $\left.D\left(A^{n+1}\right) \times D\left(A^{n}\right)\right)$, then $\left(\mathrm{ACP}_{2}\right)$ is $2 n$ - (or $2 n+1-)$ wellposed.

Proof. We prove the statement for $(x, y) \in D\left(A^{n}\right) \times D\left(A^{n}\right)$. The proof for $(x, y) \in D\left(A^{n+1}\right) \times D\left(A^{n}\right)$ requires only some modifications. By Lemma 7.2, $B=\left[\begin{array}{ll}0 & I \\ A & 0\end{array}\right]$ has nonempty resolvent set and by Lemma 
7.4, $\left(\mathrm{ACP}_{r}\right)$ has unique solutions $w(\cdot)=\left(u(\cdot), u^{\prime}(\cdot)\right)$ for all $(x, y) \in$ $D\left(B^{2 n}\right)$. Hence, by Theorem 3.1 and Lemma 7.2, there exists a locally bounded function $p(\cdot)$ such that for the once integrated solutions of $\left(\mathrm{ACP}_{r}\right)$ we have

$$
\begin{aligned}
\max & \left(\left|\int_{0}^{t} u(s) d s\right|,|u(t)-x|\right) \\
& =\left|\int_{0}^{t} w(s) d s\right| \leq q(t)|(x, y)|_{2 n-2}^{B} \\
& \leq 2 q(t)\left(|x|_{n-1}+|y|_{n-1}\right) .
\end{aligned}
$$

In the next theorem we prove that for densely defined operators $A$, exponential wellposedness of $\left(\mathrm{ACP}_{2}\right)$ is equivalent to $B=\left[\begin{array}{ll}0 & I \\ A & 0\end{array}\right]$ being the generator of an integrated semigroup on $E \times E$. For $n=1$, we will obtain from the following Theorem Sova's and Da Prato's and Giusti's as well as Kisynski's generation theorem for cosine family generators (see [6], [16], [23], [31]).

THEOREM 7.6. Let $A$ be a not necessarily densely defined linear operator on a Banach space $E$ with nonempty resolvent set and let $B$ be the operator matrix $\left[\begin{array}{ll}0 & I \\ A & 0\end{array}\right]$ on $E \times E$.

(a) If $B$ generates an $(m-1)$-times integrated semigroup on $E \times E$, then $\left(\mathrm{ACP}_{2}\right)$ is exponentially $m$-wellposed.

(b) If $A$ is densely defined and if $\left(\mathrm{ACP}_{2}\right)$ is exponentially $m$-wellposed, then $B$ generates an $(m-1)$-times integrated semigroup on $E \times E$.

Proof. (a): If $\boldsymbol{B}$ generates a $(2 n-1)$-times integrated semigroup, then, by Theorem 4.2, $\left(\mathrm{ACP}_{r}\right)$ is exponentially $(2 n, 2 n-1)$-wellposed. Hence, for $(x, y) \in D\left(B^{2 n}\right)=D\left(A^{n}\right) \times D\left(A^{n}\right)$, (ACP $\left.r\right)$ has exponentially bounded solutions $w(t)=\left(u(t), u^{\prime}(t)\right.$ ) (see Lemma 7.4). If the solutions are exponentially bounded, then the same is true for the once integrated solutions. Hence, by Theorem 3.1 and Lemma 7.2,

$$
\begin{aligned}
|u(t)-x| & \leq \max \left(\left|\int_{0}^{t} u(s) d s\right|,\left|\int_{0}^{t} u^{\prime}(s) d s\right|\right)=\left|\int_{0}^{t} w(s) d s\right| \\
& \leq C e^{w t}|(x, y)|_{2 n-2}^{B} \leq 2 C e^{w t}\left(|x|_{n-1}+|y|_{n-1}\right) .
\end{aligned}
$$

By Lemma 7.4, $u(t)$ is also the unique solution of $\left(\mathrm{ACP}_{2}\right)$. The proof of statement (a) in case that $B$ generates a $2 n$-times integrated semigroup requires only some obvious modifications.

The proof of $(b)$ is based on the following lemma. 
LEMMA 7.7. Let $A$ be a densely defined linear operator with nonempty resolvent set.

(a) If $\left(\mathrm{ACP}_{2}\right)$ is exponentially $2 n$-wellposed, then there are constants $C, w$ such that the solutions $u(\cdot)$ of $\left(\mathrm{ACP}_{2}\right)$ satisfy $\left|u^{\prime}(t)\right| \leq$ $C e^{w t}\left(|x|_{n}+|y|_{n-1}\right)$ for all $(x, y) \in D\left(A^{n}\right) \times D\left(A^{n}\right)$.

(b) If $\left(\mathrm{ACP}_{2}\right)$ is exponentially $2 n+1$-wellposed, then there are constants $C, w$ such that the solutions $u(\cdot)$ of $\left(\mathrm{ACP}_{2}\right)$ satisfy $\left|u^{\prime}(t)\right| \leq$ $C e^{w t}\left(|x|_{n}+|y|_{n}\right)$ for all $(x, y) \in D\left(A^{n+1}\right) \times D\left(A^{n}\right)$.

Proof. Let $u(\cdot)$ be the solution of $\left(\mathrm{ACP}_{2}\right)$ with initial value $(y, 0) \in$ $D\left(A^{n}\right) \times D\left(A^{n}\right)$. Then $v(t)=\int_{0}^{t} u(s) d s$ is the solution of $\left(\mathrm{ACP}_{2}\right)$ with initial value $(0, y)$. Therefore all solutions $v(\cdot)$ of $\left(\mathrm{ACP}_{2}\right)$ with initial value $(0, y) \in D\left(A^{n}\right) \times D\left(A^{n}\right)$ satisfy $\left|v^{\prime}(t)\right|=|u(t)| \leq C e^{w t}|x|_{n-1}$.

We may assume that $A^{-1}$ exists. Let $x \in D\left(A^{n+1}\right)$ and let $v(\cdot)$ be the solution of $\left(\mathrm{ACP}_{2}\right)$ with initial value $(0, A x)$. Then $w(t)=A^{-1} v^{\prime}(t)$ is a solution of $\left(\mathrm{ACP}_{2}\right)$ with initial value $(x, 0), x \in D\left(A^{n+1}\right)$ and $\left|w^{\prime}(t)\right|=|v(t)| \leq C e^{w t}|A x|_{n-1} \leq C e^{w t}|x|_{n}$.

From Theorem 7.5 we obtain that the linear operators $S(t): x \rightarrow$ $u^{\prime}(s)$ where $u(\cdot)$ is the unique solution of $\left(\mathrm{ACP}_{2}\right)$ with initial value $(x, 0) \in D\left(A^{n}\right) \times D\left(A^{n}\right)$ satisfy $|S(t) x| \leq f(t)|x|_{n}$. Therefore, the operators $S(t)$ are continuous from $\left[D\left(A^{n}\right)\right]$ into $E$. Moreover, by the considerations above, for all $x \in D\left(A^{n+1}\right)$ we have $|S(t) x| \leq C e^{w t}|x|_{n}$. But $A$ is densely defined and hence, by Lemma $2.1, D\left(A^{n+1}\right)$ is dense in $\left[D\left(A^{n}\right)\right]$. Hence $|S(t) x|=\left|u^{\prime}(t)\right| \leq C e^{w t}|x|_{n}$ for all solutions with initial value $(x, 0) \in D\left(A^{n}\right) \times D\left(A^{n}\right)$.

All solutions $v(\cdot)$ with initial value $(0, y) \in D\left(A^{n}\right) \times D\left(A^{n}\right)$ satisfy $\left|v^{\prime}(t)\right| \leq C e^{w t}|x|_{n-1}$ and all solutions of $\left(\mathrm{ACP}_{2}\right)$ with $(x, 0) \in D\left(A^{n}\right) \times$ $D\left(A^{n}\right)$ satisfy $\left|u^{\prime}(t)\right| \leq C e^{w t}|x|_{n}$. This proves statement (a). The proof of statement (b) of the lemma is similar.

Proof of Theorem 7.6 (b). Let $(x, y) \in D\left(B^{2 n}\right)=D\left(A^{n}\right) \times D\left(A^{n}\right)$ and let $u(\cdot)$ be the solution of $\left(\mathrm{ACP}_{2}\right)$. Then $w(t)=\left(u(t), u^{\prime}(t)\right)$ is a solution of $\left(\mathrm{ACP}_{r}\right)$. Using Lemmas 7.7 and 7.2 we obtain

$$
\begin{aligned}
|w(t)| & =\max \left(|u(t)|,\left|u^{\prime}(t)\right|\right) \\
& \leq C e^{w t}\left(|x|_{n}+|y|_{n-1}\right) \leq 2 C e^{w t}|(x, y)|_{2 n-1}^{B}
\end{aligned}
$$

for some $w \geq 0$. Therefore, $\left(\mathrm{ACP}_{r}\right)$ is exponentially $(2 n, 2 n-1)$ wellposed. The proof for the case that $\left(\mathrm{ACP}_{2}\right)$ has solutions for $(x, y) \in D\left(B^{2 n+1}\right)=D\left(A^{n+1}\right) \times D\left(A^{n}\right)$ requires only some obvious modifications. 
In case that $B=\left[\begin{array}{ll}0 & I \\ A & 0\end{array}\right]$ generates an $(n-1)$-times integrated semigroup on $E \times E$, it follows by (4.3) and Lemma 7.2 that, for all $(x, y) \in D\left(B^{n}\right),\left(\mathrm{ACP}_{2}\right)$ has unique solutions $v(\cdot)$ which are given by "Phragmen's representation formula"

$$
v(t)=\lim _{\mu \rightarrow \infty} \mu \sum_{k=1}^{\infty}(-1)^{k-1} \frac{1}{(k-1) !} e^{k \mu t} R\left(k^{2} \mu^{2}, A\right)[k \mu x+y] .
$$

In Theorem 7.6 (a) the assumption that $B$ generates an integrated semigroup can be weakened to the assumption that $\left(\mathrm{ACP}_{r}\right)$ is exponenetially $(m, m-1)$-wellposed. We know from Corollary 4.9 that if there are constants $M, w, k$ such that every $\mu \in C$ with $\operatorname{Re} \mu>w$ is in the resolvent set of $B$ and $|R(\mu, B)| \leq M(1+|\mu|)^{k}$ for all $\mu \in C$ with $\operatorname{Re} \mu>w$, then $\left(\mathrm{ACP}_{r}\right)$ is (at least) exponentially $(k+3, k+3)$ wellposed. If, in addition, $B$ is densely defined, then $B$ generates (at least) a $(k+3)$-times integrated semigroup. Using Lemma 7.2, one shows that $|R(\mu, B)| \leq M(1+|\mu|)^{2}\left|R\left(\mu^{2}, A\right)\right|$. Therefore we obtain the following corollary.

CoRollary 7.8. Let $A$ be a linear operator on a Banach space $E$. If there are constants $M, w, k$ such that $R\left(\mu^{2}, A\right)$ exists and satisfies $\left|R\left(\mu^{2}, A\right)\right| \leq M(1+|\mu|)^{k}$ for all $\mu \in C$ with $\operatorname{Re} \mu>w$, then $\left(\mathrm{ACP}_{2}\right)$ is exponentially wellposed.

EXAMPLE. Let $A$ be a linear operator with $\left|R\left(\mu^{2}, A\right)\right| \leq M$ for all $\mu \in C$ with $\operatorname{Re} \mu>w$. Then $|R(\mu, B)| \leq M(1+|\mu|)^{2}$ for all $\mu \in C$ with $\operatorname{Re} \mu>w$. Hence $\left(\mathrm{ACP}_{r}\right)$ is at least exponentially $(5,5)$-wellposed and, by Lemma $7.2,\left(\mathrm{ACP}_{2}\right)$ has unique solutions $u(\cdot)$ for $(x, y) \in$ $D\left(A^{3}\right) \times D\left(A^{2}\right)$ satisfying, by integrating the solutions of $\left(\mathrm{ACP}_{r}\right)$ once and applying Theorem 3.1, $|u(t)| \leq N e^{c t}\left(|x|_{2}+|y|_{2}\right)$.

In the following Corollary we show that Sova's and Da Prato and Giusti's generation theorem for strongly continuous cosine families can be easily obtained by applying Theorem 4.5 to $B=\left[\begin{array}{ll}0 & I \\ A & 0\end{array}\right]$.

COROLlary 7.9. Let $A$ be a densely defined linear operator on a Banach space $E$. Then the following statements are equivalent:

(a) $A$ has nonempty resolvent set and $\left(\mathrm{ACP}_{2}\right)$ has for all $(x, y) \in$ $D(A) \times D(A)$ a unique solution $u(\cdot)$; i.e., $A$ is the generator of a strongly continuous cosine family on $E$. 
(b) There are constants $M, w$ such that $\mu^{2}$ is in the resolvent set of $A$ whenever $\mu>w$. For every $\mu>w$ and all $k \in N_{0}$ we have

$$
\left|\frac{1}{k !} \frac{d^{k}}{d \mu^{k}}\left(\mu R\left(\mu^{2}, A\right)\right)\right| \leq M\left(\frac{1}{\mu-w}\right)^{k+1} \text {. }
$$

Proof. "(a) $\rightarrow$ (b)": From Theorem 7.6 we obtain that (a) holds if and only if $B=\left[\begin{array}{ll}0 & I \\ A & 0\end{array}\right]$ generates a once integrated semigroup on $E \times E$. In Theorem 4.5 we showed that $B$ generates a once integrated semigroup if and only if there exist $M \geq 1$ and $w>0$ such that $R(\mu, A)$ exists and satisfies

$$
\left|\frac{1}{k !} \frac{d^{k}}{d \mu^{k}}\left(\frac{1}{\mu} R(\mu, B)\right)\right| \leq M\left(\frac{1}{\mu-w}\right)^{k+1}
$$

for every $k \in N_{0}$ and $\mu>w$. By Lemma 7.2, $\mu^{2} \in \rho(A)$ if $\mu>w$ and

$$
\mu^{-1} R(\mu, B)=\left[\begin{array}{cc}
f(\mu) / \mu & f(\mu) / \mu^{2} \\
f(\mu) & f(\mu) / \mu
\end{array}\right]-\left[\begin{array}{cc}
0 & 0 \\
\mu^{-1} & 0
\end{array}\right]
$$

where $f(\mu)=\mu R\left(\mu^{2}, A\right)$. From

$$
\begin{aligned}
\left|\frac{1}{k !} \frac{d^{k}}{d \mu^{k}}\left(f(\mu)-\frac{1}{\mu}\right)\right| & \leq\left|\frac{1}{k !} \frac{d^{k}}{d \mu^{k}}\left(\mu^{1-2 n} R(\mu, B)\right)\right| \\
& \leq M\left(\frac{1}{\mu-w}\right)^{k+1} \text { and } \\
\left|1 / k ! \frac{d^{k}}{d \mu^{k}} 1 / \mu\right| \leq 1 / \mu^{k+1} & \leq M\left(\frac{1}{\mu-w}\right)^{k+1}
\end{aligned}
$$

we obtain statement (b).

"(b) $\rightarrow$ (a)": From Lemma 7.2 it follows that every $\mu>w$ is in the resolvent set of $B$. We know from Theorem 7.6 that (a) holds if and only if $B$ generates a once integrated semigroup on $E \times E$. We show that the inequality

$$
\left|\frac{1}{k !} \frac{d^{k}}{d \mu^{k}}\left(\frac{1}{\mu} R(\mu, B)\right)\right| \leq M\left(\frac{1}{\mu-w}\right)^{k+1}
$$

follows from

$$
\left|\frac{1}{k !} \frac{d^{k}}{d \mu^{k}} f(\mu)\right| \leq M\left(\frac{1}{\mu-w}\right)^{k+1} .
$$


Using the formulas

$$
\frac{d^{k}}{d \mu^{k}}[g(\mu) f(\mu)]=\sum_{j=0}^{k}\left(\begin{array}{l}
k \\
j
\end{array}\right) g^{(k-j)}(\mu) f^{(j)}(\mu)
$$

and

$$
1 / k ! \frac{d^{k}}{d \mu^{k}} 1 / \mu^{m}=(-1)^{k}\left(\begin{array}{c}
m+k-1 \\
m-1
\end{array}\right) 1 / \mu^{m+k}
$$

and (4.3) one shows easily that the inequality $(*)$ implies

$$
\left|\frac{1}{k !} \frac{d^{k}}{d \mu^{k}}\left(\mu^{-m} f(\mu)\right)\right| \leq M w^{-m}\left(\frac{1}{\mu-w}\right)^{k+1} \quad \text { for all } m \geq 0 \text {. }
$$

Hence, by (7.1) and (7.2),

$$
\left|\frac{1}{k !} \frac{d^{k}}{d \mu^{k}}\left(\frac{1}{\mu} R(\mu, B)\right)\right| \leq M\left(\frac{1}{\mu-w}\right)^{k+1} .
$$

The results of $\S 6$ can be used to obtain an "adjoint theory" for second order Cauchy problems on nonreflexive Banach spaces (on reflexive Banach spaces all statements of the previous theorems hold if and only if they are valid for the adjoint $A^{*}$ ). From Corollary 6.1 we obtain the following corollary.

COROLlary 7.10. Let $A$ be a densely defined linear operator on a nonreflexive Banach space $E$ with nonempty resolvent set. If $\left(\mathrm{ACP}_{2}\right)$ is exponentially $m$-wellposed, then the adjoint second order Cauchy problem $u^{\prime \prime}(t)=A^{*} u(t) ; u(0)=x^{*}, u^{\prime}(0)=y^{*}$ is exponentially $m+1$ wellposed on $E^{*}$.

Combining Theorem 7.6 and Corollary 5.3 we generalize Kisynski's characterization of a cosine family generator (see [16]).

COROLlary 7.11. Let $A$ be a densely defined linear operator on $a$ $B$ anach space $E$ with nonempty resolvent set and let $B$ be the operator matrix $\left[\begin{array}{ll}0 & I \\ A & 0\end{array}\right]$ on $E \times E$. Then the following statements are equivalent:

(a) $\left(\mathrm{ACP}_{2}\right)$ is exponentially $m$-wellposed.

(b) There exists a norm ||$_{F}$ on $D\left(B^{m-1}\right)$ such that $|(x, y)|$ $\leq|(x, y)|_{F} \leq C|(x, y)|_{m-1}^{B}$. The restriction of $B$ to the closure $F$ of $D\left(B^{m-1}\right)$ under the norm ||$_{F}$ generates a strongly continuous semigroup on the Banach space $F$.

8. Damped second order Cauchy problems. In this section we study damped second order Cauchy problems

$\left(\mathrm{ACP}_{d}\right) \quad u^{\prime \prime}(t)-A u^{\prime}(t)-B u(t)=0 ; \quad u(0)=x, \quad u^{\prime}(0)=y$. 
A function $u(\cdot) \in C^{2}([0, \infty), E)$ with $u(t) \in D(B)$ and $u^{\prime}(t) \in D(A)$ for all $t \geq 0$ satisfying $\left(\mathrm{ACP}_{d}\right)$ for $t \geq 0$ is called a solution of $\left(\mathrm{ACP}_{d}\right)$.

If $A$ is a semigroup generator on $E$ and if $B$ is a closed operator with $D(A) \subset D(B)$, then $\left(\mathrm{ACP}_{d}\right)$ has unique solutions $u(\cdot)$ for $(x, y) \in D(A) \times D(A)$ satisfying $|u(t)| \leq M e^{w t}\left(|x|_{1}+|y|\right)$ (see [25]). In the following, the regularity condition $D(A) \subset D(B)$ is replaced by $D\left(A^{n}\right) \subset D(B)$ for some $n>1$. As a typical example how the results of the previous sections may be applied to higher order Cauchy problems, we will give a rough analysis of

$$
\begin{aligned}
& u^{\prime \prime}(t)-A u^{\prime}(t)-\left(a A^{2}+b A+c I\right) u(t)=0 \\
& u(0)=x, \quad u^{\prime}(0)=y .
\end{aligned}
$$

For $b=c=0,(8.1)$ was introduced by Chen-Russel [5] as a mathematical model for elastic systems with structural damping.

The most natural way to study $(8.1)$ is to reduce the equation to the first order problem $w^{\prime}(t)=\Phi w(t) ; w(0)=(x, y)$ where $\Phi$ is the operator matrix on $E \times E$ given by

$$
\left[\begin{array}{cc}
0 & I \\
a A^{2}+b A+c I & A
\end{array}\right]
$$

with $D(\Phi)=D\left(A^{2}\right) \times D(A)$. Unfortunately, for a closed operator $A$, the operator $\Phi$ is in general not a closed operator on $E \times E$.

However, if $A$ has nonempty resolvent set, then, for some $\mu_{0}$ in the resolvent set of $A$, the closure $\Phi_{c}$ of $\Phi$ is given by

$$
\left[\begin{array}{cc}
\left(\mu_{0}-A\right)^{2} & 0 \\
0 & \left(\mu_{0}-A\right)^{2}
\end{array}\right] \circ\left[\begin{array}{cc}
0 & R\left(\mu_{0}, A\right)^{2} \\
\left(a A^{2}+b A+c I\right) R\left(\mu_{0}, A\right)^{2} & A R\left(\mu_{0}, A\right)^{2}
\end{array}\right]
$$

with

$$
\begin{aligned}
D\left(\Phi_{c}\right)=\{(x, y) \in E \times E & :\left(a A^{2}+b A+c I\right) \\
\times & \left.\left(R\left(\mu_{0}, A\right)^{2} x+A R\left(\mu_{0}, A\right)^{2} y\right) \in D\left(A^{2}\right)\right\} .
\end{aligned}
$$

Hence we will study (8.1) by reducing the equation to the first order problem

$$
w^{\prime}(t)=\Phi_{c} w(t) ; \quad w(0)=(x, y) .
$$

It can be easily seen that if (8.1) has a solution $u(\cdot)$, then (8.2) has a solution $w(t)=\left(u(t), u^{\prime}(t)\right)$. However, if (8.2) has a solution $w(t)=(u(t), v(t))$, then the first coordinate $u(\cdot)$ is not necessarily a solution of $(8.1)$ but only of

$$
\begin{gathered}
\begin{aligned}
& u^{\prime \prime}(t)-\left(\mu_{0}-A\right)^{2} {\left[A R\left(\mu_{0}, A\right)^{2} u^{\prime}(t)\right.} \\
&\left.+\left(a A^{2}+b A+c I\right) R\left(\mu_{0}, A\right)^{2} u(t)\right]=0 \\
& u(0)=x, \quad u^{\prime}(0)=y
\end{aligned}
\end{gathered}
$$


We will call (8.3) the closure of the Cauchy problem (8.1). (8.3) seems to be the appropriate Cauchy problem to look at if one studies (8.1) by reducing it to a first order problem in $E \times E$. Clearly, if $u(\cdot)$ is a solution of (8.1), then it is also a solution of (8.3). One should notice that a solution $u(\cdot)$ of $(8.3)$ is not necessarily in $D\left(A^{2}\right)$ and that $u^{\prime}(\cdot)$ not necessarily in $D(A)$. Hence solutions of (8.3) are not necessarily solutions of (8.1).

DEFINITION 8.1. Let $\mu_{0}$ be in the resolvent set of a linear operator $A$ on a Banach space $E$. A function $u(\cdot) \in C^{2}([0, \infty), E)$ with $A R\left(\mu_{0}, A\right)^{2} u^{\prime}(t)+\left(a A^{2}+b A+c I\right) R\left(\mu_{0}, A\right)^{2} u(t) \in D\left(A^{2}\right)$ for all $t \geq 0$ satisfying (8.3) is called a mild solution of (8.1).

It follows from the observations above that (8.1) has a (unique) mild solution if and only if (8.2) has a (unique) solution. Moreover, $w(t)$ is a solution of (8.2) if and only if $w(t)=\left(u(t), u^{\prime}(t)\right)$ where $u(t)$ is a mild solution of (8.1).

If $\Phi_{c}$ generates an $(n-1)$-times integrated semigroup on $E \times E$ (or even if (8.2) is exponentially $(n, n-1)$-wellposed), then (8.2) has unique solutions $w(t)=\left(u(t), u^{\prime}(t)\right)$ for all $(x, y) \in D\left(\Phi_{c}^{n}\right)$ which contains $D\left(A^{n+1}\right) \times D\left(A^{n}\right)$. The first coordinate function $u(t)$ is then the unique mild solution of (8.1), satisfying, by Theorem 4.5 , for large enough constants $M, w$ :

$$
\left|u^{\prime}(t)\right| \leq \max \left(|u(t)|,\left|u^{\prime}(t)\right|\right)=|w(t)| \leq M e^{w t}|(x, y)|_{n-1}^{\Phi}
$$

and

$$
|u(t)-x| \leq\left|\int_{0}^{t} w(s) d s\right| \leq M e^{w t}|(x, y)|_{n-2}^{\Phi} .
$$

If $(x, y) \in D\left(A^{n+1}\right) \times D\left(A^{n}\right) \subset D\left(\Phi_{c}^{n}\right)$, then an easy computation shows that there is a constant $C$ such that

$$
\begin{aligned}
& \left|u^{\prime}(t)\right| \leq M e^{w t}|(x, y)|_{n-1}^{\Phi} \leq C e^{w t}\left(|x|_{n}+|y|_{n-1}\right) \text { and } \\
& |u(t)| \leq M e^{w t}|(x, y)|_{n-2}^{\Phi} \leq C e^{w t}\left(|x|_{n-1}+|y|_{n-2}\right) .
\end{aligned}
$$

We summarize these observations in the following lemma.

LEMMA 8.2. If $\Phi_{c}$ generates an $(n-1)$-times integrated semigroup on $E \times E$, then (8.1) has unique mild solutions for (at least) all initial data $(x, y) \in D\left(A^{n+1}\right) \times D\left(A^{n}\right)$ which depend continuously on the initial data as described in (8.4).

To determine whether or not $\boldsymbol{\Phi}_{c}$ generates an integrated semigroup we have to determine the resolvent set of $\Phi_{c}$. An easy computation 
shows that $\mu$ is in the resolvent set of $\Phi_{c}$ if and only if $f_{i}(\mu)$ is in the resolvent set of $A(i=1,2)$, where

$$
f_{i}(\mu)=1 / 2 a\left(-\mu-b \pm\left[(1+4 a) \mu^{2}+2 b \mu+b^{2}-4 a c\right]^{1 / 2}\right) .
$$

If $\mu$ is in the resolvent set of $\Phi_{c}$, then

$$
\begin{aligned}
R\left(\mu, \Phi_{c}\right) & =\left[\begin{array}{cc}
(\mu-A) R_{\mu} & R_{\mu} \\
\left(a A^{2}+b A+c I\right) R_{\mu} & \mu R_{\mu}
\end{array}\right] \text { where } \\
\boldsymbol{R}_{\mu}= & -1 / a R\left(f_{1}(\mu), A\right) R\left(f_{2}(\mu), A\right) \\
= & g(\mu) R\left(f_{1}(\mu), A\right)-g(\mu) R\left(f_{2}(\mu), A\right)
\end{aligned}
$$

and

$$
g(\mu)=\left[(1+4 a) \mu^{2}+2 b \mu+b^{2}-4 a c\right]^{-1 / 2} .
$$

Let $H_{w}:=\left\{f_{i}(\mu) ; \mu \in C, \operatorname{Re} \mu>w\right\}$. Then it is necessary for $\Phi_{c}$ being the generator of an integrated semigroup, that there is a $w>0$ such that $H_{w}$ is contained in the resolvent set of $A$. Depending on the value of $a$, the sets $H_{w}$ have, for sufficiently large $w$, the following form

(i) If $a>0$, then, for all values of $b, c, H_{w}$ is of the form

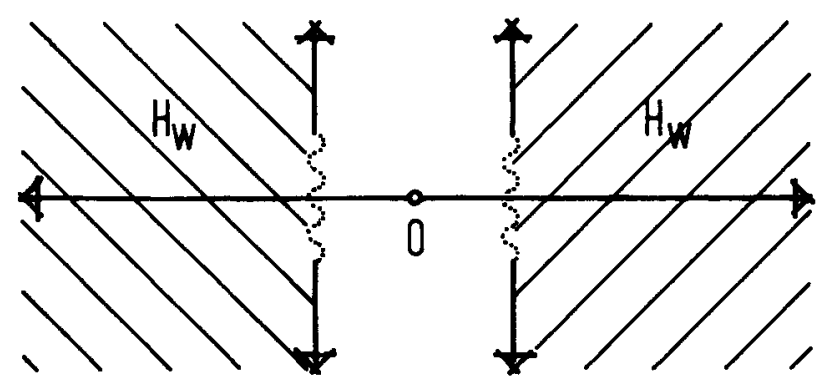

(ii) If $-0.25 \leq a \leq 0$, then, for all values of $b, c, H_{w}$ is of the form

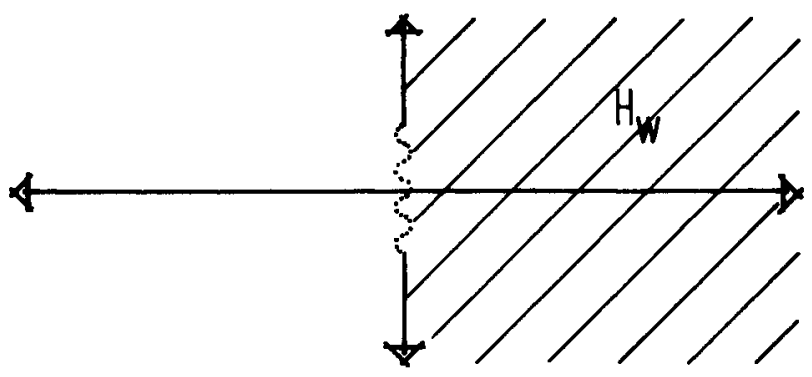


(iii) If $a<-0.25$, then, for all values of $b, c, H_{w}$ is of the form

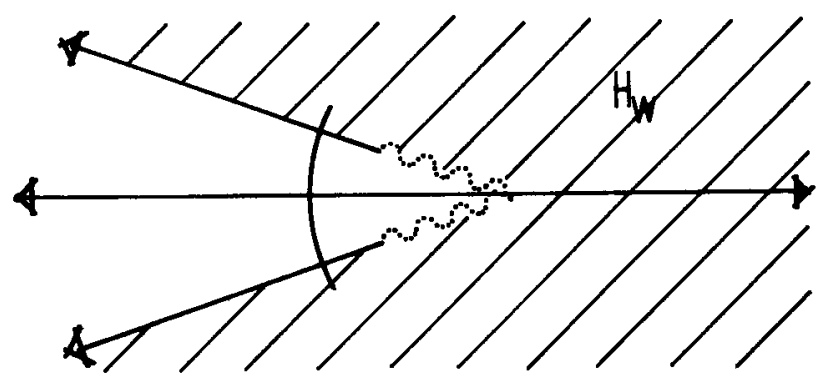

where the angle goes to zero as $a \rightarrow-\infty$.

In all cases, the intercepts of the regions $H_{w}$ with the real axis depend on values of $w$. In (I), the distance of the two parts of $H_{w}$ goes to infinity as $w \rightarrow \infty$. In (II) and (III), the region $H_{w}$ can be shifted arbitrarily to the right by increasing $w$.

If there exists a polynomial $p(\cdot)$ and a constant $w$ such that $R(\mu, A)$ exists and satisfies

$$
|R(\mu, A)| \leq p(|\mu|) \text { for all } \mu \in H_{w},
$$

then, by $(8.5)$, there exists a polynomial $q(\cdot)$ such that $\left|R\left(\mu, \Phi_{c}\right)\right| \leq$ $q(|\mu|)$ for all $\mu \in C$ with $\operatorname{Re} \mu>w$.

Therefore, by Corollary $4.9,\left(\mathrm{ACP}_{r}\right)$ is exponentially wellposed and, by Theorem 4.8 , if $A$ is densely defined, $\Phi_{c}$ is the generator of an integrated semigroup on $E \times E$. In any case, (8.6) implies that there exists an $n \in N$ (depending on the degree of the polynomial $p(\cdot)$ ) for which Lemma 8.2 holds. We summarize these observations in the following lemma.

LEMMA 8.3. If there is a $w>0$ such that $R(\mu, A)$ exists and satisfies (8.6) for every $\mu \in H_{w}$, then there exists an $n \in N$ such that, at least for all $(x, y) \in D\left(A^{n+1}\right) \times D\left(A^{n}\right)$, (8.1) has a unique mild solution $u(\cdot)$ satisfying (8.4).

As the formula (8.5) indicates one can try to use inverse Laplace transform methods to find explicit expressions of the mild solutions of (8.1) in terms of solutions of $u^{\prime}(t)=A u(t)$.

To simplify our notations we assume on that $A$ generates a strongly continuous semigroup $(T(t))$ on $E$.

Formally, for $x \in D(A)$, the solutions of (8.1) are given by the inverse Laplace transform of $\mu R(\mu) x+R(\mu)(y-A x)$, the first coordinate of $R\left(\mu, \Phi_{c}\right)(x, y)$. 
If the inverse Laplace transform of $\mu R(\mu) x$ is $H(t) x$, then the inverse Laplace transform of $\mu R(\mu) x+R(\mu)(y-A x)$ is given by $H(t) x+\int_{0}^{t} H(s)(y-A x) d s$. Formally, by (8.5),

$$
\begin{aligned}
H(t) x & =\frac{1}{2 \pi i} \int e^{\mu t} \mu g(\mu)\left[R\left(f_{1}(\mu), A\right) x-R\left(f_{2}(\mu), A\right) x\right] d \mu \\
& =\frac{1}{2 \pi i} \int e^{\mu t} \mu g(\mu)\left[\int_{0}^{\infty}\left(e^{-f_{1}(\mu) s}-e^{-f_{2}(\mu) s}\right) T(s) x d s\right] d \mu \\
& =\int_{0}^{\infty} g(t, s) T(s) x d s
\end{aligned}
$$

where

$$
g(t, s)=\frac{1}{2 \pi i} \int e^{\mu t} \mu g(\mu)\left(e^{-f_{1}(\mu) s}-e^{-f_{2}(\mu) s}\right) d \mu .
$$

LEMMA 8.4. Let $A$ be the generator of a strongly continuous semigroup on a Banach space $E$. If there is a $w \in R$ for which $H_{w}$ is contained in the resolvent set of $A$ and if the inverse Laplace transform of $\mu g(\mu)\left(e^{-f_{1}(\mu) s}-e^{-f_{2}(\mu) s}\right)$ is given by $g(t, s)$, then, for $x \in D(A)$, a generalized solution of (8.1) is given by

$$
u(t)=H(t) x+\int_{0}^{t} H(s)(y-A x) d s
$$

where $H(t) x=\int_{0}^{\infty} g(t, s) T(s) x d s$.

Because we were not able yet to find a general Laplace transform inversion formula for $\mu g(\mu)\left(e^{-f_{1}(\mu) s}-e^{-f_{2}(\mu) s}\right)$ we restrict ourself to two special cases in which the calculation of $g(t, s)$ is particularly easy.

(I) The special case of (8.1) in which $a=0$ was treated in [25]. In this case one can show that if $A$ generates a strongly continuous semigroup, then, for all $(x, y) \in D(A) \times D(A)$, there exists a unique solution $u(t)=W^{\prime}(t) x+W(t)(y-A x)$ where

$$
W(t) x:=e^{-c t} \int_{0}^{t} e^{2 b s} J_{0}\left(\sqrt{4\left(-c+b^{2}\right)(t-s) s}\right) T(s) x d s
$$

$\left(J_{0}(t)\right.$ denotes the Bessel function $\left.\sum(-1)^{n}(t / 2)^{2 n} /(n !)^{2}\right)$.

(II) If one considers (8.1) wtih $b=c=0$ and assumes that

(i) $A$ is the generator of a strongly continuous semigroup $(T(t))$ on a Banach space $E$ if $-.25 \leq a<0$,

(ii) $A$ generates a holomorphic semigroup if $a<-.25$,

(iii) $A$ generates a group if $a>0$, then, for every $x \in D\left(A^{2}\right)$ and $y \in D(A)$, there exists a solution $u(\cdot)$ 
of (8.2). The solution is given by

$$
u(t)=\frac{c_{2}}{c_{2}-c_{1}} T\left(\frac{t}{c_{2}}\right) x-\frac{c_{1}}{c_{2}-c_{1}} T\left(\frac{t}{c_{1}}\right) x+\frac{c_{1} c_{2}}{c_{2}-c_{1}} \int_{t / c_{2}}^{t / c_{1}} T(s) y d s .
$$

where $c_{i}=(-1 \pm \sqrt{1+4 a}) / 2 a$.

We show that $\Phi_{c}$ is the generator of a once integrated semigroup on $E \times E$. By the considerations above, $\mu$ is in the resolvent set of $\Phi$ if and only if the functions $f_{i}(\mu)=\mu c_{i}$ are in the resolvent set of $A$. From the assumptions (i), (ii) or (iii) it follows that there exists a $w>0$ such that every $\mu \in C$ with $\operatorname{Re} \mu>w$ is in the resolvent set of $\Phi_{c}$ and that $R\left(\mu, \Phi_{c}\right)$ is given by

$$
R\left(\mu, \Phi_{c}\right)=\left[\begin{array}{cc}
(\mu-A) R_{\mu} & R_{\mu} \\
a A^{2} R_{\mu} & \mu R_{\mu}
\end{array}\right]
$$

where $R_{\mu}=-1 / a R\left(\mu c_{1}, A\right) R\left(\mu c_{2}, A\right)$.

Using the fact that $R\left(\mu c_{i}, A\right) x$ is the Laplace transform of $\left(1 / c_{i}\right) T\left(t / c_{i}\right) x$, one can show that, for every $(x, y) \in D(A) \times D(A)$, $R(\mu, \Phi)(x, y)$ is the Laplace transform of

$$
\Gamma(t)(x, y)=\left[\begin{array}{ll}
T_{1}(t) & T_{2}(t) \\
T_{3}(t) & T_{4}(t)
\end{array}\right] \circ\left[\begin{array}{l}
x \\
y
\end{array}\right]
$$

where

$$
\begin{aligned}
& T_{1}(t)=\frac{c_{2}}{c_{2}-c_{1}} T\left(\frac{t}{c_{2}}\right)-\frac{c_{1}}{c_{2}-c_{1}} T\left(\frac{t}{c_{1}}\right), \\
& T_{2}(t)=\frac{c_{1} c_{2}}{c_{2}-c_{1}} \int_{t / c_{2}}^{t / c_{1}} T(s) d s, \\
& T_{3}(t)=\frac{1}{c_{2}-c_{1}}\left[T\left(\frac{t}{c_{2}}\right) A-T\left(\frac{t}{c_{1}}\right) A\right] \text { and } \\
& T_{4}(t)=\frac{c_{2}}{c_{2}-c_{1}} T\left(\frac{t}{c_{1}}\right)-\frac{c_{1}}{c_{2}-c_{1}} T\left(\frac{t}{c_{2}}\right) .
\end{aligned}
$$

The first coordinate $u(t)=T_{1}(t) x+T_{2}(t) y$ gives the unique mild solution of (8.1) mentioned above.

If $\Phi_{c}$ would generate a strongly continuous semigroup $V(t)$ on $E \times$ $E$, then, by the uniqueness of the Laplace transform, $\Gamma(t)(x, y)=$ $V(t)(x, y)$ for every $x, y \in D(A)$. Hence the operator family $(\Gamma(t))$ could be extended to a strongly continuous family of bounded operators on $E \times E$ which is not possible. Therefore, $\Phi_{c}$ is not a semigroup generator on $E \times E$. However, the integrated solution semigroup 
$S(t)(x, y)=\int_{0}^{t} \Gamma(s)(x, y) d s$ can be extended to a strongly continuous, exponentially bounded family in $L(E \times E)$ and $R(\mu, \Phi)(x, y)=$ $\mu \int_{0}^{\infty} e^{-\mu t} S(t)(x, y) d t$ for every $x, y \in E$. Therefore, $\Phi_{c}$ generates a once integrated semigroup on $E \times E$ and Lemma 8.2 applies with $n=2$.

9. Further examples of integrated semigroups. In this section we indicate some more possible applications of integrated semigroups. The main purpose of this chapter is not to give a detailed analysis of the examples presented, but to show that the technique of integrated semigroups might be the "right" way to treat various problems in the theory of abstract evolution equations efficiently.

EXAMPLE 9.1 ([14]). Let $E$ be one of the spaces $C_{0}(R), C_{b}(R)$, or $L^{p}(R)$ for $1 \leq p \leq \infty$ and let $A$ be defined as $A f=\sum_{i=0}^{k} a_{i} d^{i} / d x^{i}$, $a_{i} \in C$, with $D(A)=\{f: A f \in E$ in the sense of distributions $\}$. Very few of these operators define strongly continuous semigroups on the spaces $E$. In general $A$ will be not densely defined on $C_{b}$ or $L^{\infty}$; the third derivative generates a semigroup only on $L^{2}$; and on $L_{\infty}$ it is known that there is no unbounded operator at all which generates a strongly continuous semigroup (see [22], A-II-3).

However, if $\sup _{x \in R} \operatorname{Re}[p(i x)]<\infty$, where $p(x)=\sum_{i=0}^{k} a_{i} x^{i}$, then $A$ is the generator of a once integrated semigroup on the spaces $C_{0}(R)$ and $L^{p}(R)$ for $1 \leq p \leq \infty$ (see [14]).

If $E$ is one of the spaces $C_{0}\left(R^{n}\right), C_{b}\left(R^{n}\right)$, or $L^{p}\left(R^{n}\right)$ for $1 \leq p \leq \infty$, then the multidimensional results are as follows. Let $A$ be defined as $A=\sum_{|\alpha| \leq k} a_{\alpha} D^{\alpha}$ where $\alpha=\left(a_{1}, \ldots, a_{n}\right) \in N_{0}^{n},|\alpha|=a_{1}+\cdots+a_{k}$ and $D^{\alpha}=\left(d_{1} / d x_{1}\right)^{a_{1}} \ldots .\left(d_{n} / d x_{n}\right)^{a_{n}}$. Define

$$
p(x)=\sum_{|\alpha| \leq k} a_{\alpha}(-i)^{|\alpha|} x_{1}^{a_{1}} \cdots \cdot x_{n}^{a_{n}} \text { and } \operatorname{ord}(p)=\max \left(|\alpha|: a_{\alpha} \neq 0\right) \text {. }
$$

The polynomial $p(\cdot)$ is called elliptic if there are constants $L, c$ such that $|p(x)| \geq c|x|^{\operatorname{ord}(p)}$ for $|x| \geq L$. If $p(\cdot)$ is an elliptic polynomial with $\operatorname{ord}(p)>n / 2$ and if $\sup \left\{\operatorname{Re} p(x): x \in R^{n}\right\}<\infty$, then $A$ generates an $([n / 2]+2)$-times integrated semigroup on the spaces $C_{0}\left(R^{n}\right)$, $C_{b}\left(R^{n}\right)$, or $L^{p}\left(R^{n}\right)$ for $1 \leq p \leq \infty$.

As consequence one obtains that the Schroedinger operator $i \Delta$ generates at least a three times integrated semigroup on all spaces listed above if $n=2$ or $n=3$ (the Schroedinger operator is a semigroup generator only on $L^{2}$, see [9], 8.6.7; for a different, but equivalent method to study differential operators on $R^{n}$, see [3]). 
EXAMPLE 9.2. (a) If $A$ generates a semigroup of class $(0, A),(1, A)$, $\left(0, C_{1}\right),\left(1, C_{1}\right)$ or $C_{0}$ as defined in Hille-Phillips [13], $\S 10.6$, then $A$ generates a once integrated semigroups $(S(t))$ on $E$ where $S(t) x:=$ $\int_{0}^{t} T(s) x d s$.

(b) Let $A$ be the generator of a semigroup $(T(t))$ which is strongly continuous for $t>0$ and which has a singularity at 0 of growth order $\alpha$; i.e., $|T(t)| \leq M t^{-\alpha} e^{w t}$ for some $1>\alpha>0$. For a characterization of those generators $A$, see Da Prato [7] and Okazawa [27]; for applications of these semigroups, see [17] and [9]). Integrating 1-times we obtain that the operators $S(t)$ defined by $S(t) x=\int_{0}^{t} T(s) x d s$ define a 1-times integrated semigroup on $E$ and $R(\mu, A) x=\mu \int_{0}^{\infty} e^{-\mu t} S(t) x d t$ for $\operatorname{Re} \mu>w^{\prime}$. Hence generators of semigroups of growth order $\alpha$ are generators of integrated semigroups and the wellposedness results of the previous sections can be applied. As far as we know, most of the wellposedness results were not yet known for the various types of semigroups mentioned above.

EXAMPLE 9.3. It is well known that stability of a semigroup $(T(t))$ on Hilbert spaces can be characterized in terms of properties of the weak*-continuous implemented semigroup $\Gamma(t) B:=T(t) B T^{*}(t)$ on the $W^{*}$-algebra of all symmetric operators in $L(H)$ (see [22], D-IV2 ). In the following we will show that these implemented semigroups define once integrated semigroups and therefore, by Theorem 5.2, strongly continuous semigroups on an appropriate Banach space $F \subset$ $L(H)$.

The following construction works for semigroups on an arbitrary Banach space $E$ and will be used in a forthcoming paper on "Spectral theory of integrated semigroups" to characterize stability of a semigroup $(T(t))$ on $E$ (see [22], A-IV) in terms of the implemented semigroup $\Gamma(t) B:=T(t) B T(t)$ on $L(E)$.

Let $T_{1}(t), T_{2}(t)$ be two strongly continuous semigroups on a Banach space $E$ with generators $A_{1}$ and $A_{2}$. On $L(E)$ we define an operator $\Phi$ by $\Phi B=A_{1} B+B A_{2}, D(\Phi)=\left\{B \in L(E): B\left(D\left(A_{2}\right)\right) \subset D\left(A_{1}\right)\right.$ and $\Phi B$ has a bounded extension on $E$ \}. To study the $L(E)$-valued Cauchy problem $U^{\prime}(t)=\Phi U(t) ; U(0)=X \in L(E)$ we define a strongly continuous, exponentially bounded family $(S(t))$ of bounded linear operators on $L(E)$ by

$$
S(t) B: x \rightarrow \int_{0}^{t} T_{1}(s) B T_{2}(s) x d s
$$


There exists a $w>0$ such that, for every $\mu$ with $\operatorname{Re} \mu>w$ the operators $R_{\mu} B=\mu \int_{0}^{\infty} e^{-\mu t} S(t) B d t$ are well-defined bounded linear operators on $L(E)$. To show that $R_{\mu} B \in D(\Phi)$ and $(\mu-\Phi) R_{\mu} B=I$ for every $B \in L(E)$, we need the following lemma whose straightforward proof is omitted.

LEMMA 9.3.1. Let $A$ be the generator of a strongly continuous semigroup $(T(t))$ on a Banach space $E$ and let $f(\cdot) \in C^{1}\left(R^{+}, E\right)$. Then $y:=\int_{0}^{t} T(s) f(s) d s \in D(A)$ for all $t \geq 0$ and $A y=-\int_{0}^{t} T(s) f^{\prime}(s) d s+$ $T(t) f(t)-f(0)$.

Let $x \in D\left(A_{2}\right)$. Then, by the previous lemma and the closedness of the operators $A_{i}, R(\mu) B x \in D\left(A_{1}\right)$ and $A_{1} R(\mu) B x+R(\mu) B A_{2} x=$ $\mu R(\mu) B x-B x$ for all $x \in D\left(A_{2}\right)$. From the density of $D\left(A_{2}\right)$ it follows that $R_{\mu} B \in D(\Phi)$ and $(\mu-\Phi) R_{\mu} B=I$ for every $B \in L(E)$.

To show that $R_{\mu}(\mu-\Phi) B=I$ for all $B \in D(\Phi)$ take an $x \in D\left(A_{2}\right)$. By $B \in D(\Phi)$, it follows that $B D\left(A_{2}\right) \subset D\left(A_{1}\right)$ and therefore

$$
\begin{aligned}
R(\mu) \Phi B x & =\mu \int_{0}^{\infty} e^{-\mu t} \int_{0}^{t} T_{1}(s) A_{1} B T_{2}(s) x+T_{1}(s) B A_{2} T_{2}(s) x d s d t \\
& =\mu \int_{0}^{\infty} e^{-\mu t}\left[T_{1}(t) B T_{2}(t) x-B x\right] d t=\mu R(\mu) \Phi B x-B x
\end{aligned}
$$

Hence every $\mu$ with $\operatorname{Re} \mu>w$ is in the resolvent set of $\Phi$ and $R(\mu, \Phi)=R_{\mu}$. Therefore $\Phi$ is the generator of a once integrated semigroup on $L(E)$ and our wellposedness results can be applied. Especially, by Theorem $5.2, \Phi$ is the generator of a strongly continuous semigroup on the closure of $D\left(\Phi^{2}\right) \subset\{B \in L(E): S(\cdot) B \in$ $\left.C^{2}\left(R^{+}, L(E)\right)\right\}$ under the norm ||$_{F}:|B|_{F}=\sup _{t \geq 0}\left|e^{-w t} S^{\prime}(t) B\right|$.

In general, the operator $\Phi$ is not densely defined. However, it can be easily seen that $\mu R_{\mu} B x \rightarrow B x$ as $\mu \rightarrow \infty$ for every $B \in L(E)$ and every $x \in E$. Therefore $D(\Phi)$ is dense in $L(E)$ with respect to the topology of strong convergence.

It would be interesting to investigate in how far the following construction can be used to develop a stability theory for nonautonomous evolution equations which uses in a canonical way the stability results for the autonomous equation.

Let $U(t, s)$ be a strongly continuous evolution operator defined by a wellposed nonautonomous Cauchy problem

$$
u^{\prime}(t)=A(t) u(t), \quad u(s)=x, \quad t \geq s
$$


satisfying $|U(t, s)| \leq M$ for all $t \geq s$. Let $L$ be the Banach space $L^{\infty}\left(R^{+}, L(E)\right)$. On $L$ we define a semigroup $T(t)$ by $T(t) f(s)=$ $f(t+s) U(t+s, s)$. It is obvious that stability properties of the semigroup $(T(t))$ translate into stability properties for the evolution family $U(t, s)$.

In general, $(T(t))$ is not strongly continuous on $L$. However, the once integrated semigroup $S(t) f(s)=\int_{0}^{t} f(r+s) U(r+s, s) d r$ is strongly continuous on $L$. Let $A f(\cdot)=f^{\prime}(\cdot)+f(\cdot) A(\cdot)$. Then every $\mu>0$ is in the resolvent set of $A$ and $R(\mu, A) f=\mu \int_{0}^{\infty} e^{-\mu t} S(t) f d t$. Therefore $A$ is the generator of the once integrated semigroup $(S(t))$ on $L$.

To develop a stability theory along the lines mentioned above one would have to investigate in how far spectral properties of the generator $A$ (which will depend on properties of the operators $A(t)$ ) translate into stability properties for $T(t) f$ and hence into stability properties for the evolution family $U(t, s)$.

Acknowledgment. I would like to thank W. Arendt and H. Kellermann for many helpful and stimulating discussions.

\section{REFERENCES}

[1] W. Arendt, Resolvent positive operators, Proc. London Math. Soc., (3) 54 (1987), 321-349.

[2] - Vector valued Laplace transforms and Cauchy problems, Israel J. Math., (3), 54 (1987), 327-352.

[3] M. Balabane and H. A. Emamirad, Smooth distribution group and Schrödinger equation in $L^{p}\left(R^{n}\right)$, J. Math. Anal. Appl., 70 No. 1, (1979), 61-71.

[4] R. Beals, On the abstract Cauchy problem, Funct. Anal., 10 (1972), 281-299.

[5] G. Chen and D. L. Russel, $A$ mathematical model for linear elastic systems with structural damping, Quarterly Applied Math., January 1982, 433-454.

[6] G. Da Prato and E. Giusti, Una catterizzarione dei generatori di funzioni coseno astratto, Boll. Unione Mat. Ital., 22 (1967), 357-362.

[7] G. Da Prato, Semigruppi di crescenza n, Ann. Scuola Norm. Super. Pisa Sci. Fis. Mat., 20, No. 4, (1966), 753-782.

[8] E. B. Davies and M. M. Pang, The Cauchy problem and a generalization of the Hille-Yosida Theorem, preprint 1986.

[9] H. O. Fattorini, The Cauchy Problem, Addison Wesley, Reading, MA, 1983.

[10] - Second Order Linear Differential Equations in Banach Spaces, NorthHolland Mathematics Studies 108, North-Holland, (1985).

[11] J. A. Goldstein, Semigroups of Linear Operators and Applications, Oxford University Press, New York, (1985).

[12] J. Hadamard, Lectures on Cauchy's Problem in Linear Partial Differential Equations, Yale University Press, New Haven, 1923.

[13] E. Hille and R. S. Phillips, Functional Analysis and Semigroups, Amer. Math. Soc. Providence, Rhode Island 1957.

[14] H. Kellermann and M. Hieber, Integrated semigroups, J. Funct. Anal., to appear. 
[15] J. Kisynski, Semigroups of operators and some of their applications to partial differential equations, in: Control Theory and Topics in Functional Analysis, Vol. III, IAEA, Vienna, 1976.

[16] - On cosine operator functions and one-parameter groups of operators, Studia Math., 44 (1972), 93-105.

[17] S. G. Krein and M. I. Khazan, Differential equations in a Banach space, J. Soviet Math., 30 (1985), 2154-2239.

[18] S. G. Krein, Linear Differential Equations in Banach Spaces, Amer. Math. Soc. Transl. 29, Providence (R. I.) 1971.

[19] J. Lagnese, On equations of evolution and parabolic equations of higher order in $t$, J. Math. Anal. Appl., 32 No. 1 (1970), 15-37.

[20] J. L. Lions, Les semi-groupes distributions, Portugal. Math. 19 (1960), 141-164.

[21] I. Miyadera, S. Oharu, and N. Okazawa, Generation theorems of semigroups of linear operators, Publs. Res. Inst. Math. Sci., Kyoto Univ. 8, No. 3, (1973), 509-555.

[22] R. Nagel (ed.), One-parameter Semigroups of Positive Operators, Lect. Notes Math., 1184 (1986), Springer.

[23] F. Neubrander, Well-posedness of abstract Cauchy problems, Semigroup Forum, 29 (1984), 74-85.

[24] - On the relation between the semigroup and its infinitesimal generator, Proc. Amer. Math. Soc., 99, No. 4, 1987.

[25] - Well-posedness of higher order abstract Cauchy problems, Trans. Amer. Math. Soc., 295 (1986), 257-290.

[26] S. Oharu, Semigroups of linear operators in a Banach space, Publ. RIMS, Kyoto Univ., 7 (1971/72), 205-260.

[27] N. Okazawa, A generation theorem for semigroups of growth order $\alpha$, Tohoku Math. J., 26 No. 1, (1974), 39-51.

[28] A. Pazy, Semigroups of Linear Operators and Applications to Partial Differential Equations, Springer, New York 1983.

[29] N. Sanekata, Some remarks on the abstract Cauchy problem, Publ. RIMS, Kyoto Univ., 11 (1975), 51-65.

[30] M. Sova, Problèmes de Cauchy paraboliques abstraits de classes superieures et les semi-groupes distributions, Ricerche Mat., 18 (1969), 215-238.

[31] _ Cosine operator functions, Rozprawy Math., 49 (1966), 1-46.

[32] T. Takenaka and N. Okazawa, Abstract Cauchy problems for second order linear differential equations in a Banach space, preprint, (1986).

Received April 9, 1987 and in revised form July 27, 1987. This research was supported by the National Science Foundation through Grant DMS-8601983. 



\section{PACIFIC JOURNAL OF MATHEMATICS}

\section{EDITORS}

\author{
V. S. VARADARAJAN \\ (Managing Editor) \\ University of California \\ Los Angeles, CA 90024 \\ HeRBERT ClEMENS \\ University of Utah \\ Salt Lake City, UT 84112 \\ R. FINN \\ Stanford University \\ Stanford, CA 94305
}

\section{HERMANN FLASCHKA \\ University of Arizona \\ Tucson, AZ 85721}

VAUghan F. R. Jones

University of California

Berkeley, CA 94720

ROBION KIRBY

University of California

Berkeley, CA 94720

\author{
C. C. MOORE \\ University of California \\ Berkeley, CA 94720
}

HAROLD STARK

University of California, San Diego

La Jolla, CA 92093

\section{ASSOCIATE EDITORS}
R. ARENS
E. F. BECKENBACH
B. H. NEUMANN
F. WOLF
K. YOSHIDA
(1906-1982)

\section{SUPPORTING INSTITUTIONS}

\begin{abstract}
UNIVERSITY OF ARIZONA
UNIVERSITY OF BRITISH COLUMBIA

UNIVERSITY OF CALIFORNIA

MONTANA STATE UNIVERSITY

UNIVERSITY OF NEVADA, RENO

NEW MEXICO STATE UNIVERSITY

OREGON STATE UNIVERSITY
\end{abstract}
CALIFORNIA INSTITUTE OF TECHNOLOGY

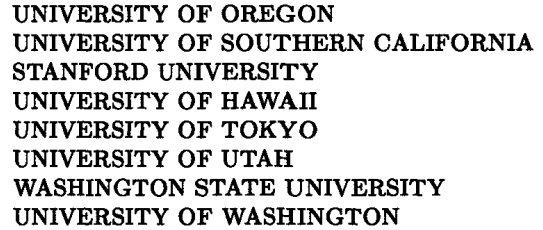

The Supporting Institutions listed above contribute to the cost of publication of this Journal, but they are not owners or publishers and have no responsibility for its content or policies.

Mathematical papers intended for publication in the Pacific Journal of Mathematics should be in typed form or offset-reproduced (not dittoed), double spaced with large margins. Please do not use built up fractions in the text of the manuscript. However, you may use them in the displayed equations. Underline Greek letters in red, German in green, and script in blue. The first paragraph must be capable of being used separately as a synopsis of the entire paper. In particular it should contain no bibliographic references. Please propose a heading for the odd numbered pages of less than 35 characters. Manuscripts, in triplicate, may be sent to any one of the editors. Please classify according to the scheme of Math. Reviews, Index to Vol. 39. Supply name and address of author to whom proofs should be sent. All other communications should be addressed to the managing editor, or Elaine Barth, University of California, Los Angeles, California 90024.

There are page-charges associated with articles appearing in the Pacific Journal of Mathematics. These charges are expected to be paid by the author's University, Government Agency or Company. If the author or authors do not have access to such Institutional support these charges are waived. Single authors will receive $\mathbf{5 0}$ free reprints; joint authors will receive a total of 100 free reprints. Additional copies may be obtained at cost in multiples of 50 .

The Pacific Journal of Mathematics is issued monthly as of January 1966. Regular subscription rate: $\$ 190.00$ a year (5 Vols., 10 issues). Special rate: $\$ 95.00$ a year to individual members of supporting institutions.

Subscriptions, orders for numbers issued in the last three calendar years, and changes of address should be sent to Pacific Journal of Mathematics, P.O. Box 969, Carmel Valley, CA 93924, U.S.A. Old back numbers obtainable from Kraus Periodicals Co., Route 100, Millwood, NY 10546.

The Pacific Journal of Mathematics at P.O. Box 969, Carmel Valley, CA 93924 (ISSN 0030-8730) publishes 5 volumes per year. Application to mail at Second-class postage rates is pending at Carmel Valley, California, and additional mailing offices. Postmaster: send address changes to Pacific Journal of Mathematics, P.O. Box 969, Carmel Valley, CA 93924.

PUBLISHED BY PACIFIC JOURNAL OF MATHEMATICS, A NON-PROFIT CORPORATION Copyright (C) 1988 by Pacific Journal of Mathematics 


\section{Pacific Journal of Mathematics}

Vol. 135, No. $1 \quad$ September, 1988

Margaret M. Bayer, Barycentric subdivisions $\ldots \ldots \ldots \ldots \ldots \ldots \ldots \ldots \ldots$

Eung Chun Cho, $s$-Smith equivalent representations of dihedral groups . . . . 17

Avner Friedman and Jindrich Necas, Systems of nonlinear wave equations

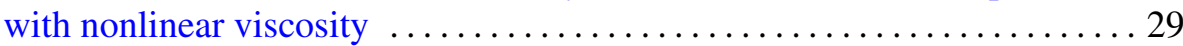

Kyong Taik Hahn, Nontangential limit theorems for normal mappings . . . . 557

Eloise A. Hamann, Evan Green Houston, Jr. and Jon Lee Johnson,

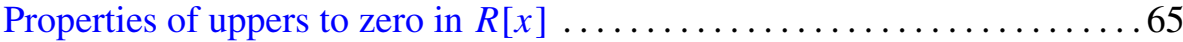

José M. Souto Menéndez, On a theorem due to Cassels ............. 81

Courtney Hughes Moen, Irreducibility of unitary principal series for

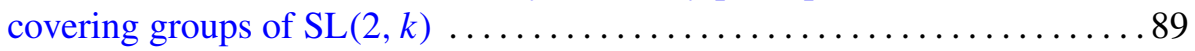

Frank M. Neubrander, Integrated semigroups and their applications to the

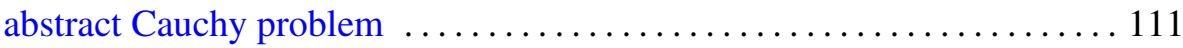

C. K. Qu and Roderick Sue-Chuen Wong, Szegő's conjecture on Lebesgue

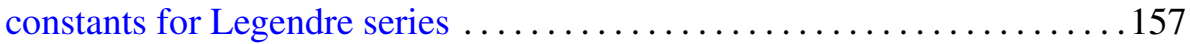

John Brendan Sullivan, The Euler character and cancellation theorems for

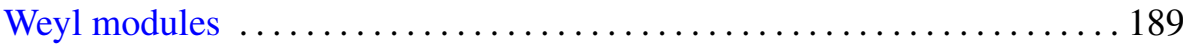

